\title{
AN ABUNDANCE ANALYSIS FOR FOUR RED HORIZONTAL-BRANCH STARS IN THE EXTREMELY METAL-RICH GLOBULAR CLUSTER NGC $6528^{1}$
}

\author{
Eugenio Carretta, ${ }^{2}$ Judith G. Cohen, ${ }^{3}$ Raffaele G. Gratton, ${ }^{2}$ and Bradford B. Behr ${ }^{3,4}$ \\ Received 2000 March 1; accepted 2001 June 6
}

\begin{abstract}
We present the results of the first analysis of high-dispersion spectra of four red horizontal-branch stars in the metal-rich globular cluster NGC 6528, located in Baade's window. We find that the mean $[\mathrm{Fe} / \mathrm{H}]$ for NGC 6528 is $+0.07 \pm 0.01$ dex (error of the mean), with a star-to-star scatter of $\sigma=0.02$ dex (four stars), although the total error is likely to be larger $(\sim 0.1 \mathrm{dex})$ because of systematic errors related to the effective temperature scale and to model atmospheres. This metallicity is somewhat larger than both the mean abundance in the galactic bulge found by McWilliam \& Rich (1994) and that found in our previous paper for NGC 6553. However, we find that the spectra of clump stars in NGC 6528 and 6553 are very similar each other, the slightly different metal abundances found being possibly due to the different atmospheric parameters adopted in the two analyses. Since the present analysis is based on higher quality material, we propose to revise our previous published metal abundance for NGC 6553 to $[\mathrm{Fe} / \mathrm{H}]=-0.06 \pm 0.15$. For NGC 6528 , we find excesses for the $\alpha$-process elements $\mathrm{Si}$ and $\mathrm{Ca}$ $([\mathrm{Si} / \mathrm{Fe}]=+0.4$ and $[\mathrm{Ca} / \mathrm{Fe}]=+0.2)$, whereas $\mathrm{Mn}$ is found to be underabundant $([\mathrm{Mn} / \mathrm{Fe}]=-0.4)$. We find a solar abundance of $\mathrm{O}$; however, this is somewhat uncertain because of the dependence of the $\mathrm{O}$ abundance on the adopted atmospheric parameters and the coupling between $\mathrm{C}$ and $\mathrm{O}$ abundances in these cool, metal-rich stars. Finally, we find large $\mathrm{Na}$ excesses $([\mathrm{Na} / \mathrm{Fe}] \sim+0.4)$ in all stars examined.

Key words: globular clusters: general - globular clusters: individual (NGC 6528) - stars: abundances

On-line material: machine-readable table
\end{abstract}

\section{INTRODUCTION}

The bulge is one of the major components of the Milky Way. Its integrated properties resemble those of giant elliptical galaxies, which in turn are one of the dominant components of the universe. However, in contrast to these distant environments, the relative proximity of the galactic bulge allows us to study individual stars in detail and (at least in some cases) derive accurate abundances for the local version of old metal-rich populations. This capability is very helpful in reconstructing the history of formation, still quite controversial, of these very important constituents of the universe.

Unfortunately, owing to the rather large distance and the substantial interstellar absorption characteristic of the bulge, without a significant boost from microlensing, detailed abundance analyses of bulge stars have to be limited to the evolved population. An exploratory study of field bulge stars was carried out by McWilliam \& Rich (1994); coupled with other studies at lower resolution (Rich 1988), this study indicated that the bulge has a wide abundance distribution, peaking at a metal abundance (as represented by $[\mathrm{Fe} / \mathrm{H}]$ ) slightly lower than solar and may have

\footnotetext{
${ }^{1}$ Based in large part on observations obtained at the W. M. Keck Observatory, which is operated operated as a scientific partnership among the California Institute of Technology, the University of California, and the National Aeronautics and Space Administration (NASA). The Observatory was made possible by the generous financial support of the W. M. Keck Foundation.

2 Osservatorio Astronomico di Padova, Vicolo dell'Osservatorio 5, I-35122 Padova, Italy.

${ }^{3}$ Palomar Observatory, Mail Stop 105-24, California Institute of Technology, Pasadena, CA 91125.

4 Current address: Department of Astronomy, University of Texas, Austin, TX 78712.
}

an excess of $\alpha$-elements. The latter would suggest that most of the bulge formed within a rather short interval of time because of the interplay between star formation and the lifetime of the progenitors responsible for the synthesis of different elements.

While this analysis is of great value, the interpretation of the results is complicated by the fact that we are not able to determine the ages of the stars observed. Thus, for example, from these data alone, we are unable to deduce any strong constraint on the epoch of formation of the bulge. This concern may, at least in principle, be overcome by studying stars in clusters. The bulge of our Galaxy has a rich population of globular clusters (Ortolani 1999). The colormagnitude diagrams (CMDs) of these clusters indicate that several of them are metal-rich, (quite similar to the bulk of the field bulge population), and they are likely old (Ortolani et al. 1995). However, accurate metal abundances are needed to determine ages with the precision required to understand whether the bulge is as old as (or even older than) the halo or whether instead it has the somewhat younger age of the oldest stars in the thin disk. Thus, accurate abundance determinations for bulge globular clusters represent a basic step in our understanding the formation of the Milky Way.

Such an analysis also provides additional important pieces of information, since it allows us to (1) extend the calibration of abundance scales at high metal abundance, poorly known at present, since metal-rich clusters tend to be concentrated toward the galactic center, where reddening and crowding often hamper accurate observations; (2) derive quite accurate reddening estimates by comparing the observed colors with those expected for stars having temperatures determined from spectroscopically derived parameters, such as line excitation, which are independent of reddening; and (3) further constrain the evolution of the 
bulge by determining the ratios of the abundances of $\alpha$ elements to iron (crucial to determining the rate of chemical evolution).

In order to address these questions, in a previous study (Cohen et al. 1999, henceforth Paper I), we used the Keck telescope to acquire high-resolution spectra of individual stars in NGC 6553. The large aperture of the Keck telescope and the efficiency of its high resolution spectrograph (HIRES; Vogt et al. 1994) allowed us to observe stars on the red horizontal branch (RHB). As explained in Paper I, this choice is clearly advantageous as compared with observations of first ascent red giants, since RHB stars are warmer, making an abundance analysis of their spectra much easier. Furthermore, contamination of the RHB region of the CMD by field stars is much less important than in the case of red giants, resulting in a higher probability of membership (as confirmed a posteriori by the measured radial velocities). In Paper I, we found that NGC 6553 has a metallicity of $[\mathrm{Fe} / \mathrm{H}]=-0.16 \pm 0.08$, somewhat higher than determined by the analysis of two cool giants by Barbuy et al. (1999, henceforth B99) and very similar to the bulk of bulge field giants observed by McWilliam \& Rich (1994). In addition, we found an excess of the $\alpha$-elements similar to that found for the field stars in the bulge.

In the present paper, we perform for the first time a similar study on NGC 6528, a globular cluster having values of the metallicity indicators very similar to those of NGC 6553 (Harris 1996). NGC 6528 is a very highly concentrated cluster, between us and the galactic bulge, at about $7.8 \mathrm{kpc}$ from the Sun, hence very close to the galactic center (see, e.g., Ortolani et al. 1995). Located in Baade's window with $(l, b)=(1.1,-4.2)$, it seems certain that NGC 6528 is a bulge cluster. Moreover, the cluster velocity indicates that it is not a disk cluster. Ortolani et al. (1995) found from careful comparison of both CMDs and luminosity functions that its stellar population is very similar to that of NGC 6553 and of Baade's window. In addition, recently Davidge (1999), noting that stars in NGC 6528 populate the same region in the two-color plane $(J-H, H-K)$ of the field bulge giants, supported the classification of this cluster as a true bulge cluster, rather than a very metal-rich disk cluster.
The metallicity of NGC 6528 is uncertain. Zinn \& West (1984) derived an abundance of +0.2 dex, but Armandroff \& Zinn (1988), using an analysis of the Ca IR triplet in the integrated cluster spectrum, decreased that value significantly to -0.23 dex. Other methods, such as that of Sarajedini (1994), cannot be applied because of the lack of calibrating clusters in the high-metallicity regime.

NGC 6553 and 6528 are the only very high metallicity globular clusters that can be studied rather easily at optical wavelengths; the remaining bulge clusters are either more metal-poor or very obscured. Hence, the choice of NGC 6528 for the present study was obvious. While the analysis presented here is very similar to that already done on NGC 6553 , it is based on higher signal-to-noise ratio $(\mathrm{S} / \mathrm{N})$ spectra; moreover, we adjusted the instrumental setup in order to include lines of $\mathrm{Na}$, as well as $\mathrm{O}$. This was deemed important because observations of $\mathrm{O}$ and $\mathrm{Na}$ in very metalrich clusters may help to better understand the pattern of abundances of these elements found in more metal-poor globular clusters (Ivans et al. 1999; Kraft et al. 1998). In fact, serious doubts were cast on the up-to-now most favored mechanism (deep mixing) by Gratton et al. (2001), who found that the O-Na anticorrelation (previously seen only for stars on the RGB of globular clusters) is clearly present among dwarf, unevolved stars in NGC 6752. On the other hand, the alternative explanation suggested (pollution due to mass lost by previous AGB stars in the cluster) is expected to be sensitive to the overall metal abundance of the cluster (Ventura, D'Antona, \& Gratton 2001). Determination of O abundances in NGC 6528 RHB stars is favored by the large radial velocity of this cluster, which shifts stellar lines away from telluric lines.

\section{OBSERVATIONS AND REDUCTION}

\subsection{Choice of Program Stars}

Program stars were selected using high-resolution images and VIJK photometry of NGC 6528 from $H S T$ (VI) and IRAC2 $(J K)$ kindly provided by P. Montegriffo (1999, private communication). Table 1 gives the most relevant parameters of these stars, while Figure 1 shows their location within the cluster and Figure 2 the position of these objects in the $V-(V-I)$ CMD of NGC 6528. In Figure 1,

TABLE 1

SAMPLE OF STARS ObSERVED IN NGC 6528

\begin{tabular}{|c|c|c|c|c|c|c|c|c|c|}
\hline $\begin{array}{l}\text { ID } \\
(1)\end{array}$ & $\begin{array}{c}V^{\mathrm{a}} \\
(\mathrm{mag}) \\
(2)\end{array}$ & $\begin{array}{c}I^{\mathrm{a}} \\
(\mathrm{mag}) \\
(3)\end{array}$ & $\begin{array}{c}J^{\mathrm{a}} \\
(\mathrm{mag}) \\
(4)\end{array}$ & $\begin{array}{c}K^{\mathrm{a}} \\
(\mathrm{mag}) \\
(5)\end{array}$ & $\begin{array}{c}v_{r}^{\mathrm{b}} \\
\left(\mathrm{km} \mathrm{s}^{-1}\right) \\
(6)\end{array}$ & $\begin{array}{l}\text { Date of Observation } \\
\text { (7) }\end{array}$ & $\begin{array}{c}\text { Exposure Time } \\
\text { (s) } \\
(8)\end{array}$ & $\begin{array}{l}\text { R.A. (J2000) } \\
\qquad(9)\end{array}$ & $\begin{array}{c}\text { Decl. (J2000) } \\
\text { (10) }\end{array}$ \\
\hline $5422 \ldots \ldots$ & 16.95 & 15.34 & 14.11 & 13.14 & $\begin{array}{l}206.8 \pm 1.0 \pm 2.5 \\
206.4 \pm 0.7 \pm 1.5\end{array}$ & $\begin{array}{l}1999 \text { Aug } 17 \\
2000 \text { June } 3\end{array}$ & $\begin{array}{l}2 \times 1200 \\
6 \times 1200\end{array}$ & 180447.58 & -300330 \\
\hline $3025 \ldots \ldots$ & 17.14 & 15.43 & 14.21 & 13.22 & $\begin{array}{l}218.1 \pm 1.0 \pm 2.5 \\
216.8 \pm 0.7 \pm 1.5\end{array}$ & $\begin{array}{l}1999 \text { Aug } 17 \\
2000 \text { Jun } 3\end{array}$ & $\begin{array}{r}3 \times 1200 \\
12 \times 1200\end{array}$ & 180447.62 & -300336 \\
\hline $3046 \ldots \ldots$ & 17.25 & 15.50 & 14.12 & 13.34 & $207.3 \pm 1.0 \pm 2.5$ & 1999 Aug 18 & $2 \times 1200$ & 180451.52 & -300320 \\
\hline $5425 \ldots \ldots$ & 17.09 & 15.35 & 14.25 & 13.32 & $209.2 \pm 0.8 \pm 2.5$ & 2000 Jun 3 & $<2500$ & 180447.62 & -300325 \\
\hline $3032^{c} \ldots \ldots$ & 16.69 & 14.71 & 13.58 & 12.34 & $211.2 \pm 0.8 \pm 2.5$ & 2000 Jun 3 & $<2500$ & 180447.34 & -300333 \\
\hline
\end{tabular}

NoTE.- Units of right ascension are hours, minutes, and seconds, and units of declination are degrees, arcminutes, and arcseconds.

${ }^{\text {a }}$ From P. Montegriffo (1999, private communication).

$\mathrm{b}$ The internal error in $v_{r}$ is followed by the systematic error.

${ }^{c}$ This star is a red giant in NGC 6528 not an RHB star. 


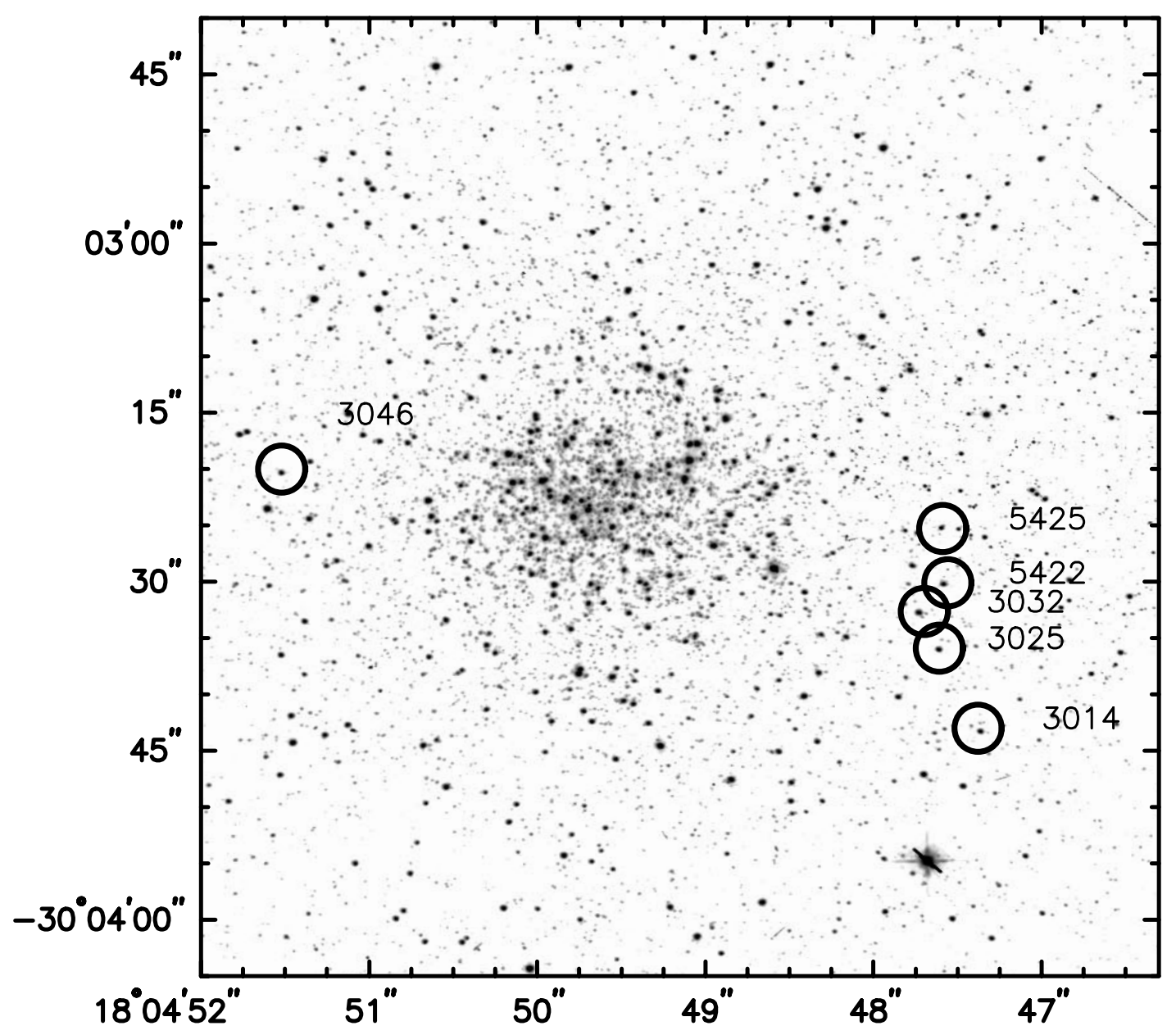

FIG. 1. - Stars in NGC 6528 studied here are marked on this subset from a $100 \mathrm{~s}$ WFPC2 image from the HST archive taken with the F555W filter

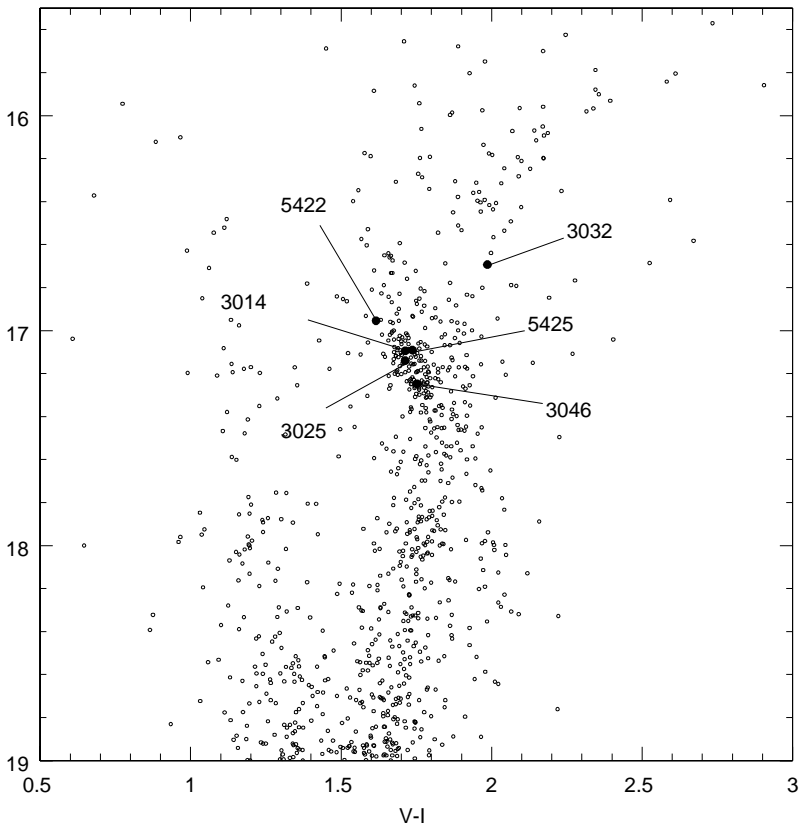

FIG. 2.-NGC $6528 V-(V-I)$ CMD. Stars studied in the present work are indicated with large filled circles and are labeled in the figure. Note that stars 3032 and 5425 were not used in the abundance analysis because of the low $\mathrm{S} / \mathrm{N}$ of their spectra. the stars studied here are marked on this subset from a $100 \mathrm{~s}$ WFPC2 image from the HST archive. ${ }^{5}$ Coordinates for the program stars are given in Table 1 . The field of the cluster is very crowded. We selected for observation a number of apparently uncrowded stars having colors and magnitudes appropriate for the RHB in the CMD in order to maximize the probability of cluster membership. Beside the four program stars (namely, 5422, 3014, 3025, and 3046), two other stars fell in the edge of the slit (stars 3032 and 5425) on at least some of the spectra. While not good enough for abundance analysis, spectra of these extra stars are adequate for precise radial velocities. A posteriori, membership of the program stars in NGC 6528 was confirmed by their radial velocity: this is a very useful criterion in this case because of the large $v_{r}$ of this cluster; according to Harris (1996), the heliocentric $v_{r}$ is $184.9 \pm 3.8$ (internal error) $\mathrm{km}$ $\mathrm{s}^{-1}$, and its $v_{r}$ relative to the solar local standard of rest is $195 \mathrm{~km} \mathrm{~s}^{-1}$. Heliocentric $v_{r}$ for the program stars as measured on our spectra are given in the column (6) of Table 1: the average heliocentric $v_{r}$ from our spectra is $209.9 \pm 1.6$ $\mathrm{km} \mathrm{s}^{-1}$ with $\sigma=4.0 \mathrm{~km} \mathrm{~s}^{-1}$ from six stars, giving double

${ }^{5}$ Obtained from the data archive at the Space Telescope Science Institute (STScI). STScI is operated by the Association of Universities for Research in Astronomy, Inc., under NASA contract NAS 5-26555. 
weight to velocity from the 2000 June spectra that have a higher $\mathrm{S} / \mathrm{N}$ ratio.

The radial velocities were measured by cross-correlating the region $6130-6170 \AA$ in echelle order 57 and the region 6240-6280 $\AA$ in echelle order 58, using the 2000 June spectrum of star 3025 as a template. These measurements were carried out independently and with different software packages by E. C. and J. C. The zero point was determined by fitting Gaussians to 16 lines in these orders in the spectrum of the template judged to be unblended based on their FWHM. The laboratory wavelengths of these lines were taken from the NIST Atomic Spectra Database. (NIST Standard Reference Database No. 78). The agreement between orders is excellent and confirms the dispersion solution from the ThAr lamp.

The internal radial velocity errors were calculated following the method of Tonry \& Davis (1979) using the relation $\sigma\left(v_{r}\right)=\alpha /[1+R(\mathrm{TD})]$, where the parameter $R(\mathrm{TD})$ is a measure of the ratio of the height of the peak of the crosscorrelation to the noise in the cross-correlation function away from the peak. The constant $\alpha$ was set at $15 \mathrm{~km} \mathrm{~s}^{-1}$, which represents a value typical of those found in other recent HIRES programs using similar instrumental configurations by Mateo et al. (1998), Cook et al. (1999), and Côté et al. (1999). The maximum error in $v_{r}$ incurred by a point source that is on the edge of the $1^{\prime \prime}$ slit compared with an object that uniformly fills the slit is $4 \mathrm{~km} \mathrm{~s}^{-1}$, and this factor will be smaller for real stars under real seeing conditions that are partially in the slit. The systematic errors are thus generously set at $1.5 \mathrm{~km} \mathrm{~s}^{-1}$ for the program stars observed in 2000 , and $2.5 \mathrm{~km} \mathrm{~s}^{-1}$ for the stars observed in 1999, as well as for those that fortuitously appeared in the slits.

Our velocity dispersion measured for NGC 6528 is by far the smallest published for this cluster and is consistent with a normal mass-to-light ratio for NGC 6528 . However, our mean $v_{r}$ is distinctly higher than the literature value in the compilation by Harris. A search of the references quoted by Harris revealed that all low values of $v_{r}$ for NGC 6528 are from older studies, while the most recent works tend to give a value quite similar to our own. For example, Minniti (1995) found an average $v_{r}$ of $203 \pm 20 \mathrm{~km} \mathrm{~s}^{-1}$ from seven stars, noting also that the $v_{r}$ of this cluster was found to have some discrepancy in previous analyses (e.g., in Armandroff \& Zinn 1988). The recent extensive work by Rutledge et al. (1997b) found $v_{r}=212.2 \mathrm{~km} \mathrm{~s}^{-1}$ with $\sigma=13.5 \mathrm{~km} \mathrm{~s}^{-1}$ (external error) based on eight stars. Both these results are in very good agreement with our $v_{r}$. We note that for NGC 6553, Rutledge et al. (1997b) found $v_{r}=8.4 \mathrm{~km} \mathrm{~s}^{-1}(\sigma=8.4$ $\mathrm{km} \mathrm{s}^{-1}$ ), in quite good agreement with the value of Paper I. Moreover, Rutledge et al. (1997b) already noted that their high value for NGC 6528 was different at the $3.4 \sigma$ level from the mean value in Harris' compilation and expressed concern about the potential impact on measurements of $v_{r}$ of possible nonmembers in clusters so heavily contaminated by field stars. This discussion further supports our adopted strategy of selecting target objects among the RHB stars.

The confirmation of this high $v_{r}$ is important, since it is among the largest in absolute value for a bulge cluster so close to the galactic center. The radial velocity of NGC 6528 $\left(209.9 \pm 1.6 \mathrm{~km} \mathrm{~s}^{-1}\right)$ might be a reflection of the velocity dispersion of the metal-rich bulge globular cluster population (Côté 1999), although it appears as rather extreme for a bulge object: in fact, this value is slightly less than $2 \sigma$ (of the scatter of values for individual field stars) from the mean value for bulge K giants (Terndrup, Sadler, \& Rich 1995), M giants (Sharples, Walker, \& Cropper 1990), RR Lyraes (Gratton 1987), and Miras (Feast, Robertson, \& Black 1980 ). While this large radial velocity clearly rules out the possibility that NGC 6528 is a disk cluster, there is the possibility that it belongs to the inner halo rather than to the bulge, and its orbit is by chance just passing through the bulge at the present time. The distinction between inner halo and bulge is not clearly defined at present. To obtain better insight into this issue, we examined more closely the large sample of bulge $\mathrm{K}$ giants located in Baade's window, as is NGC 6528, observed by Terndrup et al. We will consider only those stars fainter than $V=16$ (contamination by disk interlopers being important for brighter objects). For these stars, Terndrup et al. found an average velocity of $-8 \pm 6 \mathrm{~km} \mathrm{~s}^{-1}$, with a dispersion of $110 \pm 10$ for individual objects. Twenty out of 334 stars (that is, $6 \%$ ) have radial velocities in excess of $200 \mathrm{~km} \mathrm{~s}^{-1}$ (in absolute value), i.e., have kinematics similar to or more extreme than that of NGC 6528. The average metallicity for these stars (Sadler, Rich, \& Terndrup 1996) is $[\mathrm{Fe} / \mathrm{H}]=-0.17 \pm 0.12$, with a scatter for individual stars of 0.53 dex. The average value is not significantly different from the average metal abundance they found for the whole sample of bulge $\mathrm{K}$ giants $([\mathrm{Fe} / \mathrm{H}]=-0.11 \pm 0.04)$. These stars are clearly much more metal-rich than the traditional value for halo stars $([\mathrm{Fe} / \mathrm{H}]<-1)$ and have a metal abundance quite similar to that found by us for NGC 6528. Note that these considerations are based on the high radial velocity of NGC 6528 , which makes its membership in the disk population unlikely. On the other hand, the low radial velocity of NGC 6553 is compatible with that of a disk object. Some support for this view is given by a recent study by Beaulieu et al. (2001). Their CMD for NGC 6553 does not show a good match to the field bulge population and favors a metallicity comparable to solar, in good agreement with the revised value that we suggest in the present work (see below).

\subsection{Details of the Observations}

Observations were carried out with HIRES at Keck I. The HIRES detector is not large enough to yield full spectral coverage in a single exposure. The RHB stars in NGC 6528 are faint, and hence the exposures are long, so a single compromise instrumental configuration is mandatory. In our first effort described in Paper I, we wished to avoid crowding of lines and hence centered the spectra rather far toward the red. It turned out that, ignoring $\mathrm{Fe} I$ absorption lines, there were few useful features beyond $8000 \AA$, and also that line crowding was tolerable even at the blue end of the HIRES spectra of the RHB stars in NGC 6553. Thus, for the observations of the NGC 6528 RHB stars, the instrumental configuration was set to shift the spectra blueward, so that the $\mathrm{O}$ triplet at $7770 \AA$ appears in the reddest order included. This configuration had the advantage of adding features of several important elements with no lines in the wavelength regime covered in Paper I, such as $\mathrm{Na}$, as well as important additional $\mathrm{Fe}$ II lines, to improve the analysis of ionization equilibrium. However, the downside of this layout was that the unblended $\mathrm{Mg}$ I line at $8717 \AA$ was lost, while the $\mathrm{Mg}$ I lines included in this setup are more blended and more saturated.

These fields are rather crowded. Given the freedom to rotate the slit to a desired position angle and the ability to track at a fixed position angle, in many cases it might be 
possible to get two or more candidate RHB stars within the length of the HIRES slit. At the time of our initial observations for NGC 6553, HIRES did not have an image rotator to compensate for the rotation of the field at the Nasmyth focus, and hence only one star could be observed at a time. However, by 1999, the HIRES image rotator had been completed by David Tytler and the Lick Observatory engineering group (D. Tytler 2000, private communication). The maximum slit length that can be used with our instrumental configuration without overlapping echelle orders is $14^{\prime \prime}$. Leaving room at the ends of the slit for sky, we therefore searched the list of RHB candidates for suitable pairs of RHB candidates located not more than 7" apart.

The observations were carried out in two runs 10 months apart:

1. Run of 1999 August. Because of a hardware problem, the lower dome shutter of the Keck I telescope was not movable, and at low elevations, it vignetted the telescope. This forced us to observe only one object in NGC 6528 per night and that only during the hour centered on culmination of the cluster.

Exposures were $1200 \mathrm{~s}$, and three such were obtained for stars 3014 and 3025 , while only two were obtained from 3046 and 5422 . The stars were dithered by $1 "$ or $2^{\prime \prime}$ along the length of the slit between exposures.

2. Run of 2000 June. Two exposures were obtained in NGC 6528 on the nights of 2000 June 2 and 3 (i.e., one each night). Each was $7200 \mathrm{~s}$ long, in $1200 \mathrm{~s}$ segments, with small spatial dithers along the slit between each segment. Each exposure contained the RHB star 3025 and a second star, either 3014 or 5422 . Thus, the total exposure for star 3025 was $14,400 \mathrm{~s}$. The HIRES setup was identical for these two exposures. The $\mathrm{S} / \mathrm{N}$ of these spectra, based strictly on the count rate in the continuum near the center of the echelle orders, is 65 pixel $^{-1}$ (130 per 4 pixel resolution element) for star 3025 and 95 per 4 pixel resolution element for the other two stars in NGC 6528.

The spectra from both runs were reduced using the suite of routines for analyzing echelle spectra written by McCarthy (1988) within the FIGARO image processing package (Shortridge 1988). The stellar data are flat-fielded with quartz lamp spectra, thereby removing most of the blaze profile, and the results are normalized to unity by fitting a 10th-order polynomial to line-free regions of the spectrum in each order.

\subsection{Measurement of Equivalent Widths}

The spectra of the three stars observed in 2000 June (stars 3014,3025 , and 5422) are of high $\mathrm{S} / \mathrm{N}$, and equivalent widths were measured directly from them; the 1999 August spectra were not used in the abundance analysis for these objects. However, star 3046 was only observed in 1999 . The $\mathrm{S} / \mathrm{N}$ of the 1999 spectra is not very large (typically $\sim 30$ ). For this star only, we followed the same procedure successfully used in Paper I to improve the reliability of measures of equivalent widths. We filtered the spectra by convolving them with a Gaussian having a FWHM of $0.3 \AA$ (this reduced the resolution of the spectra down to $\sim 15,000$ but enhanced the $\mathrm{S} / \mathrm{N}$ per pixel to about 60 ). We also applied the above procedure to the spectra of the three other stars of the 1999 run in order to derive a linear relationship between the two sets of measured EWs and thus to correct the $1999 \mathrm{EWs}$ of star 3046, measured using convolved spectra, to that of the higher precision 2000 June spectra:

$$
\operatorname{EW}(2000)=0.90 \mathrm{EW}(1999)+12.5,
$$

with the correlation coefficient $r=0.93$ and $\sigma=19.7$ from 361 lines.

Since the spectra of the program stars are very line rich, the following procedure was adopted to measure reliable EWs on these spectra. First, we corrected the continuum slightly by using a spline interpolating function: appropriate "continuum" points were selected by comparing the spectra of different stars, so that the fiducial continuum level was set consistently for each star. This was done for all program stars. However, for star 3046, whose spectra are at a somewhat lower resolution, greater care is needed. We then selected a small number of clean lines and used these lines to set a fiducial relation between the FWHM of lines and their EWs. EWs for a larger number of lines were then measured by using a special fitting routine that measures EWs using this relation between FWHM and EWs, shown in Figure 3. With this scheme, the FWHM, which is very sensitive to the presence of blending lines, was constrained when measuring the EWs of individual lines, resulting in much more stable measures.

Note that for each line, the fitting routine used an interactively selected portion of the line profile, so that regions of the profile obviously disturbed by blending lines were not considered. In this way, we are not using just the central pixel, but a portion of the line profile that is generally broader than the FHWM of the line (by itself broader than a resolution element), and we are fitting the central part of the line adopting a model of the line profile that is a Gaussian, whose FWHM is a (linear) function of the EW. As also noted by the referee, it is, however, possible that EWs are somewhat overestimated. On the other side, since the continuum is likely to be somewhat underestimated, if the two effects are not mutually compensating, we should notice some trends of abundances as a function of the wavelength, and this is not the case.

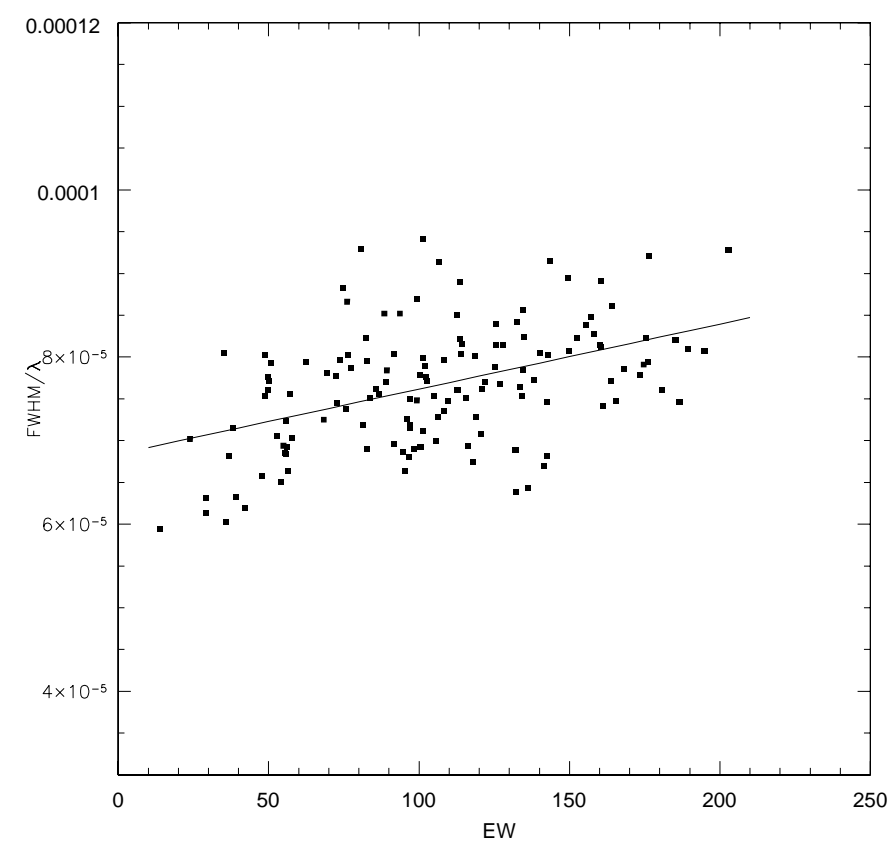

FIG. 3.-Fiducial relation between the FWHM of lines and their EWs for star 3046, observed at lower resolution. 
Apart from errors due to the continuum placement and to blends, random errors, $\delta \mathrm{EW}$, in these EWs are $\delta \mathrm{EW}=\mathrm{FWHM} /[\sqrt{n}(\mathrm{~S} / \mathrm{N})]$, where $n$ is the number of points used in the fitting, and $\mathrm{S} / \mathrm{N}$ is per pixel. In our case, $n \sim 1.06 \mathrm{FWHM} / d x$ (actually somewhat larger, in general), where $d x$ is the wavelength step; that is, we may write $\delta \mathrm{EW}=1.06 \mathrm{FWHM} /\left(\mathrm{S} / \mathrm{N}^{*}\right)$, where now $\mathrm{S} / \mathrm{N}^{*}$ is the $\mathrm{S} / \mathrm{N}$ per FWHM element (generally larger than a resolution element).

Table 2 lists the final values of the EWs, along with the adopted $g f$-values. Errors in the EWs measured by this procedure may be estimated by comparing the EWs of different stars, since all four RHB stars have very similar atmospheric parameters (see below). Through such comparisons, we obtain typical rms scatters about the linear relationship between two stars of $9 \mathrm{~m} \AA$. If we assume that both sets of EWs have equal errors, we can estimate that typical errors in EWs are $6 \mathrm{~mA}$ from the better quality spectra of 2000 June. These errors are mainly due to uncertainties in the positioning of the fiducial continuum. Errors in EWs measured on previous 1999 spectra (i.e., namely for star 3046) are somewhat larger, between 10 and $12 \mathrm{~m} \AA$.

\section{ABUNDANCE ANALYSIS}

\subsection{Determination of Atmospheric Parameters}

The analysis of the CMD of NGC 6528 (Ortolani, Bica, \& Barbuy 1992) revealed that the interstellar reddening toward this cluster is quite large, is somewhat uncertain, and has strong variations even within small projected distances on the sky (see the Appendix). Infrared photometry by Cohen \& Sleeper (1995) confirmed the presence of substantial reddening variations within this cluster. For this reason, it is not possible to derive accurate atmospheric parameters from the observed colors of stars in NGC 6528. Following the approach adopted in Paper I (where we had a similar problem), effective temperatures for the program stars were derived directly from the spectra by forcing $\mathrm{Fe}$ I lines of different excitation to provide the same abundance (typically 90-100 Fe I lines were measured for each star, 70 for star 3046, with lower quality spectra). The errors on the linear regression fits allow one to estimate the (internal) errors in these temperatures: they are $\pm 85 \mathrm{~K}$ (corresponding to $1 \sigma \mathrm{rms}$ uncertainty of $0.017 \mathrm{dex} \mathrm{eV}^{-1}$ in the slope). As an example, the left panels of Figure 4 show the run of abundances from individual $\mathrm{Fe}$ I lines with excitation potential for the RHB stars. Systematic errors mainly depend on the set of model atmospheres used in the analysis (Kurucz 1993a; models with overshooting, for consistency with the analysis of Carretta \& Gratton 1997). They are not easy to estimate, but we feel they are about $\pm 100 \mathrm{~K}$.

Observation at slightly shorter wavelengths than done in Paper I allowed a larger number of Fe II lines to be measured (typically at least four good Fe II lines for each star). We were then able to estimate surface gravities, $\log g$, from the equilibrium of ionization of $\mathrm{Fe}$. Internal errors in these surface gravities are \pm 0.36 dex (where we considered both the contributions due to errors in EWs of individual lines and those due to uncertainties in the adopted $T_{\text {eff }}$ ). Again systematic errors are mainly related to the adopted model atmospheres and to the assumption of LTE made throughout this paper. (This seems a solid assumption for the stars we are currently considering; see Gratton et al. 1999.) The average gravity we derived $(2.16 \pm 0.14)$ is very close to that predicted by evolutionary models for RHB stars in such a metal-rich cluster (about 2.3 dex, from the latest Padova isochrones; Salasnich et al. 2000), supporting the temperature scale adopted in the present analysis.

Microturbulent velocities $v_{t}$ were derived by eliminating any trend in the derived abundances from $\mathrm{Fe}$ I lines with expected equivalent widths for the lines (following the approach of Magain 1984). Given the large number of Fe I lines measured, internal error bars in these $v_{t}$ 's are small $\left( \pm 0.12 \mathrm{~km} \mathrm{~s}^{-1}\right)$. As an example, the central panels of Figure 4 show the run of abundances from individual $\mathrm{Fe} \mathrm{I}$ lines with EWs for the four program stars.

Of course, it is well known that in abundance analyses the derived values of microturbulent velocity depend on the $g f$-values one is using. Our $g f$-values are obtained by combining mainly two sources (see Carretta \& Gratton 1997, henceforth CG97, for detailed references): (1) for all strong lines and a few of the weak ones, we are using laboratory $g f$-values from a compilation of data from the literature. Only $g f$-values with errors less than 0.05 dex were considered. (2) For the vast majority of weak lines (most of the lines measurable in the spectra of NGC 6528 stars), we use solar $g f$-values, obtained from an inverse analysis, whose zero point is set by the lines having laboratory $g f$-values (and whose abundance generally agree well with meteoritic values).

As noted also by the referee, there is some correlation between line strength and excitation potential $(\chi)$, in the sense that there is a generic tendency of low-excitation $\mathrm{Fe} \mathbf{I}$ lines to be stronger than those of high excitation. This is

TABLE 2

Equivalent Widths For Four Red HB StaRs IN NGC $6528^{\mathrm{ab}}$

\begin{tabular}{cccccccc}
\hline \hline Ion & $\begin{array}{c}\lambda \\
(\AA)\end{array}$ & $\begin{array}{c}\chi \\
(\mathrm{eV})\end{array}$ & \multicolumn{1}{c}{$\log g f$} & $\begin{array}{c}5422 \\
(\mathrm{~m} \AA)\end{array}$ & $\begin{array}{c}3025 \\
(\mathrm{~m} \AA)\end{array}$ & $\begin{array}{c}3014 \\
(\mathrm{~m} \AA)\end{array}$ & $\begin{array}{c}3046 \\
(\mathrm{~m} \AA)\end{array}$ \\
\hline O I $\ldots \ldots$. & 6300.31 & 0.00 & -9.75 & 40.0 & 39.0 & 37.0 & $\ldots$ \\
O I $\ldots \ldots$. & 6363.79 & 0.02 & -10.25 & 9.0 & 15.0 & 13.0 & $\ldots$ \\
O I $\ldots . .$. & 7771.95 & 9.11 & 0.33 & 40.0 & 36.0 & 35.0 & 54.8 \\
O I $\ldots . .$. & 7775.40 & 9.11 & -0.03 & 30.0 & 31.0 & $\ldots$ & $\ldots$ \\
\hline
\end{tabular}

Table 2 is presented in its entirety in the electronic edition of the Astronomical Journal. A portion is shown here for guidance regarding its form and content.

a As described in the text, all $W_{\lambda}$ are measured from original spectra, but for star 3046 they are measured on convolved spectra and converted to the system of the other $W_{\lambda}$.

${ }^{\mathrm{b}} \mathrm{Fe} \mathrm{I}$ lines marked with an asterisk are those adopted as very clean lines; see text in $\S 3.3$. 

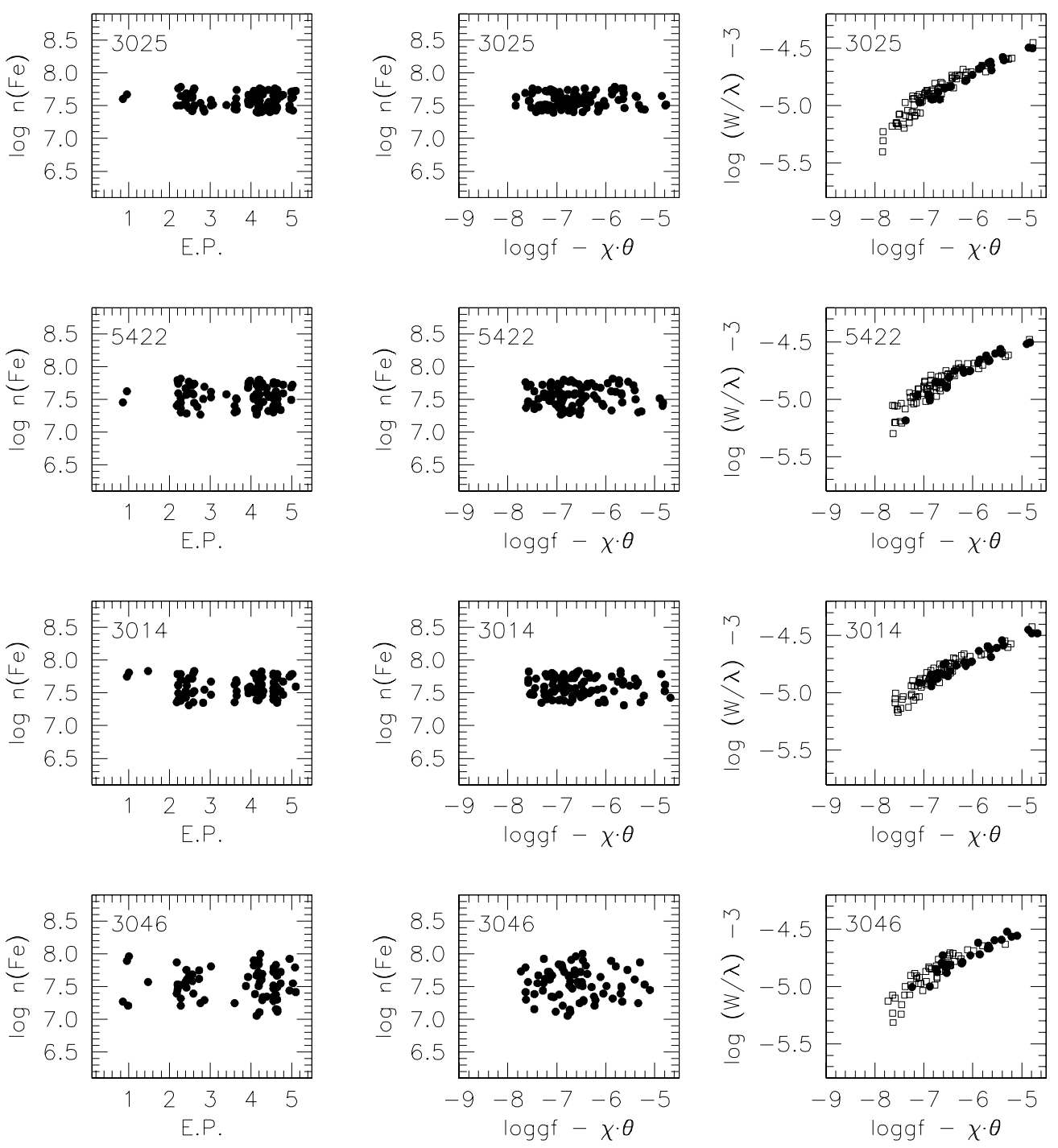

FIG. 4.-Abundances deduced from neutral Fe I lines as a function of excitation potential of the lower level of the transition (left) and of the EWs (middle) for the NGC 6528 RHB stars. In the panels on the right, curves of growth are also displayed, with open squares for lines with $\chi>3 \mathrm{eV}$ and filled circles for lines having $\chi \leq 3 \mathrm{eV}$.

shown by the curves of growth in the right-hand panels of Figure 4, in which open squares are used for lines with $\chi>3 \mathrm{eV}$, while filled circles are for lines with $\chi \leq 3 \mathrm{eV}$.

To test the relevance of this problem in our analysis, we repeated it, considering only those $\mathrm{Fe}$ I lines having $\chi>3$ $\mathrm{eV}$; we did not changed the temperature, since the excitation range included is now too small to allow fitting of $T_{\text {eff }}$, but we reoptimized the microturbulent velocity. When only lines with $\chi>3 \mathrm{eV}$ are considered, we are essentially using only solar $g f$-values; these lines are weak in the solar spectrum, so that these $g f$-values are virtually independent of details in the solar analysis. The $v_{t}$ values we derived from this subset of lines are smaller than the original ones by $0.07 \pm 0.06 \mathrm{~km} \mathrm{~s}^{-1}$, and the $\mathrm{Fe}$ abundances are larger by $0.03 \pm 0.03$ dex. Both these values are barely significant and much lower than the other sources of errors. The final adopted atmospheric parameters are shown in Table 3.

An analysis of the influence of errors on the derived abundances is given in Table 4. This table was obtained in the conventional way, specifically by comparing the abun- dances derived for star 3025 with those derived by varying the atmospheric parameters, one at a time, by the amount given in Table 4. As expected, larger effects arise from uncertainties in $T_{\text {eff }}$ (in particular for neutral species) and in gravity (in particular for singly ionized species), whereas the overall metal abundance and the microturbulent velocity only play a minor role. The last column of Table 4 gives the quadratic sum of effects due to the individual parameters

TABLE 3

\begin{tabular}{cccc}
\hline \hline Star ID & $\begin{array}{c}T_{\text {eff }} \\
(\mathrm{K})\end{array}$ & $\begin{array}{c}\log g \\
(\mathrm{dex})\end{array}$ & $\begin{array}{c}v_{t} \\
\left(\mathrm{~km} \mathrm{~s}^{-1}\right)\end{array}$ \\
\hline RHB Stars: & & & \\
$5422 \ldots \ldots$ & 4580 & 2.3 & 1.25 \\
$3025 \ldots \ldots$ & 4650 & 2.3 & 1.32 \\
$3014 \ldots \ldots$ & 4630 & 2.0 & 1.45 \\
$3046 \ldots \ldots$ & 4580 & 2.0 & 1.34 \\
\hline
\end{tabular}


TABLE 4

SEnsitivity of Abundances to Atmospheric Parameters

\begin{tabular}{crcccc}
\hline \hline Ratio & $\begin{array}{c}\Delta T_{\text {eff }} \\
(+100 \mathrm{~K})\end{array}$ & $\begin{array}{c}\Delta \log g \\
(+0.2 \mathrm{dex})\end{array}$ & $\begin{array}{c}\Delta[\mathrm{A} / \mathrm{H}] \\
(+0.1 \mathrm{dex})\end{array}$ & $\begin{array}{c}\Delta v_{t} \\
\left(+0.2 \mathrm{~km} \mathrm{~s}^{-1}\right)\end{array}$ & Total \\
\hline$[\mathrm{O} / \mathrm{Fe}] \ldots \ldots \ldots$ & -0.352 & -0.131 & -0.142 & -0.073 & 0.41 \\
{$[\mathrm{Na} / \mathrm{Fe}] \ldots \ldots$.} & 0.000 & -0.058 & -0.030 & -0.003 & 0.06 \\
{$[\mathrm{Mg} / \mathrm{Fe}] \ldots \ldots$.} & 0.050 & -0.078 & 0.037 & 0.047 & 0.11 \\
{$[\mathrm{Al} / \mathrm{Fe}] \ldots \ldots$.} & -0.125 & -0.078 & -0.063 & -0.013 & 0.16 \\
{$[\mathrm{Si} / \mathrm{Fe}] \ldots \ldots \ldots$} & -0.100 & 0.019 & 0.008 & 0.045 & 0.11 \\
{$[\mathrm{Ca} / \mathrm{Fe}] \ldots \ldots$.} & 0.062 & -0.040 & -0.021 & -0.035 & 0.08 \\
{$[\mathrm{Sc} / \mathrm{Fe} \mathrm{II]} \ldots \ldots$.} & 0.123 & -0.031 & -0.009 & -0.008 & 0.13 \\
{$[\mathrm{Ti} / \mathrm{Fe} \mathrm{I}] \ldots \ldots$.} & 0.134 & -0.016 & -0.018 & -0.014 & 0.14 \\
{$[\mathrm{~V} / \mathrm{Fe}] \ldots \ldots \ldots$} & 0.128 & -0.013 & -0.028 & -0.039 & 0.14 \\
{$[\mathrm{Cr} / \mathrm{Fe}] \ldots \ldots$.} & 0.083 & -0.012 & -0.013 & 0.029 & 0.09 \\
{$[\mathrm{Mn} / \mathrm{Fe}] \ldots \ldots$.} & 0.037 & -0.028 & -0.005 & -0.033 & 0.06 \\
{$[\mathrm{Fe} / \mathrm{H} \mathrm{I}] \ldots \ldots$.} & 0.050 & 0.018 & 0.013 & -0.087 & 0.10 \\
{$[\mathrm{Fe} / \mathrm{H} \mathrm{II}] \ldots \ldots$.} & -0.123 & 0.111 & 0.042 & -0.062 & 0.18 \\
{$[\mathrm{Ni} / \mathrm{Fe}] \ldots \ldots$.} & -0.027 & 0.034 & 0.008 & -0.001 & 0.04 \\
{$[\mathrm{Ba} / \mathrm{Fe} \mathrm{II}] \ldots \ldots$.} & -0.050 & 0.042 & 0.020 & -0.093 & 0.12 \\
\hline
\end{tabular}

listed; this can be taken as an estimate of the total uncertainty due to errors in atmospheric parameters. The uncertainty in $\mathrm{O} / \mathrm{Fe}$ is by far the largest entry in this column.

\subsection{Results}

One of our goals is to extend the calibration of the metallicity scale for globular clusters of Carretta \& Gratton (1997) to the metal-rich regime. Therefore, for the sake of consistency, we adopt the same atomic parameters (listed in Table 2) that they used, just as we did in Paper I. Note that the only difference in the line lists used for NGC 6528 and 6553 is in the slightly different wavelength region covered because of the different instrumental configuration of HIRES used for the observations. However, both lists are simply subsets of that used in CG97, securing the required homogeneity. In addition, the same set of model atmospheres (Kurucz 1993a; with convective overshooting), code for abundance analysis, etc., previously adopted are also used in the present work. Combining our previous work on NGC 6553 (Cohen et al. 1999) with the present study doubles the sample of clusters with metal abundances near the solar value, while retaining a highly homogeneous fine abundance analysis for all clusters studied to date.

From the four RHB stars, we find that the mean $[\mathrm{Fe} / \mathrm{H}]$ for NGC 6528 is $+0.07 \pm 0.01$ dex, with a star-to-star scatter of $\sigma=0.02$ dex. This is the first high-dispersion analysis of a galactic globular cluster in which an abundance greater than solar has been obtained. Note, however, that the uncertainties are from statistics only. A fair estimate of the total error bar should also include systematic errors, which are, in general, rather hard to quantify: including systematics, a conservative budget could be about $0.1 \mathrm{dex}$, mostly due to errors related to the temperature scale and the adopted model atmospheres.

The resulting abundances for each species in each star are listed in Table 5. As in Paper I, all element ratios are computed with respect to $\mathrm{Fe}$ I, except for $\mathrm{O}$ I and $\mathrm{Sc}$ II, for which we used abundances from $\mathrm{Fe}$ II to minimize the uncertainties resulting from the choice of $T_{\text {eff }}$. The rms dispersion in abundance among the measured set of lines for each ion is given in parentheses, and the adopted solar abundances are shown in the last column of Table 5. The results for star
3046 are from EWs converted to the same system of the three other stars. Only abundances derived from at least two lines are shown for star 3046. In addition, for comparison, we give in the last column of this table the abundances obtained by a similar analysis of the well-known Population I star $\zeta$ Cyg from Paper I.

For oxygen and sodium (discussed in detail below), we also give the abundances (from line analysis), including corrections, for departures from LTE, following Gratton et al. (1999).

Abundances for Sc II, V I, and Mn I include detailed corrections for the quite large hyperfine structure of their lines (see Gratton 1989; Gratton \& Sneden 1991, for references).

The $\mathrm{Ca}$ abundances were derived, applying to each line used the collisional damping parameter appropriate for that line (Smith \& Raggett 1981).

An estimate of Eu abundance was obtained by comparing the average spectrum of all the RHB stars in NGC 6528 observed in the region of the Eu II line at $6645.11 \AA$ with synthetic spectra (Fig. 5). From this comparison, evidence for a mild $([\mathrm{Eu} / \mathrm{Fe}] \simeq 0.2)$ overabundance of $\mathrm{Eu}$ is found.

\subsection{Checks on the Derived Metal Abundances}

A potential problem affecting the reliability of our results could be the contamination of $\mathrm{Fe} \mathrm{I}$ lines by blends. McWilliam \& Rich (1994) demonstrated that in bulge stars the effects of $\mathrm{CN}$ lines alone can be overwhelming at metallicities whose mean is similar to the values derived here. We expect this concern to be much less important in our spectra, since they have a much higher resolution than those used by McWilliam \& Rich, and the temperature of the stars is higher. However, in order to check the impact of this problem, we considered the Fe I lines in more detail. For each $\mathrm{Fe} \mathrm{I}$ line included, we synthesized a spectral region of $3.2 \AA$ centered on the line, using line lists extracted from the Kurucz CD-ROMs (CD-ROM 23 [Kurucz \& Bell 1995] for the atomic lines and CD-ROM 15 [Kurucz 1993b] for diatomic molecules; note that these lists include lines due to $\mathrm{CN}$, as well as to other molecules) and the same model atmospheres used for the program stars. (In this exercise, we assumed $[\mathrm{C} / \mathrm{Fe}]=-0.5$ and $[\mathrm{N} / \mathrm{Fe}]=+0.5$ since, as 


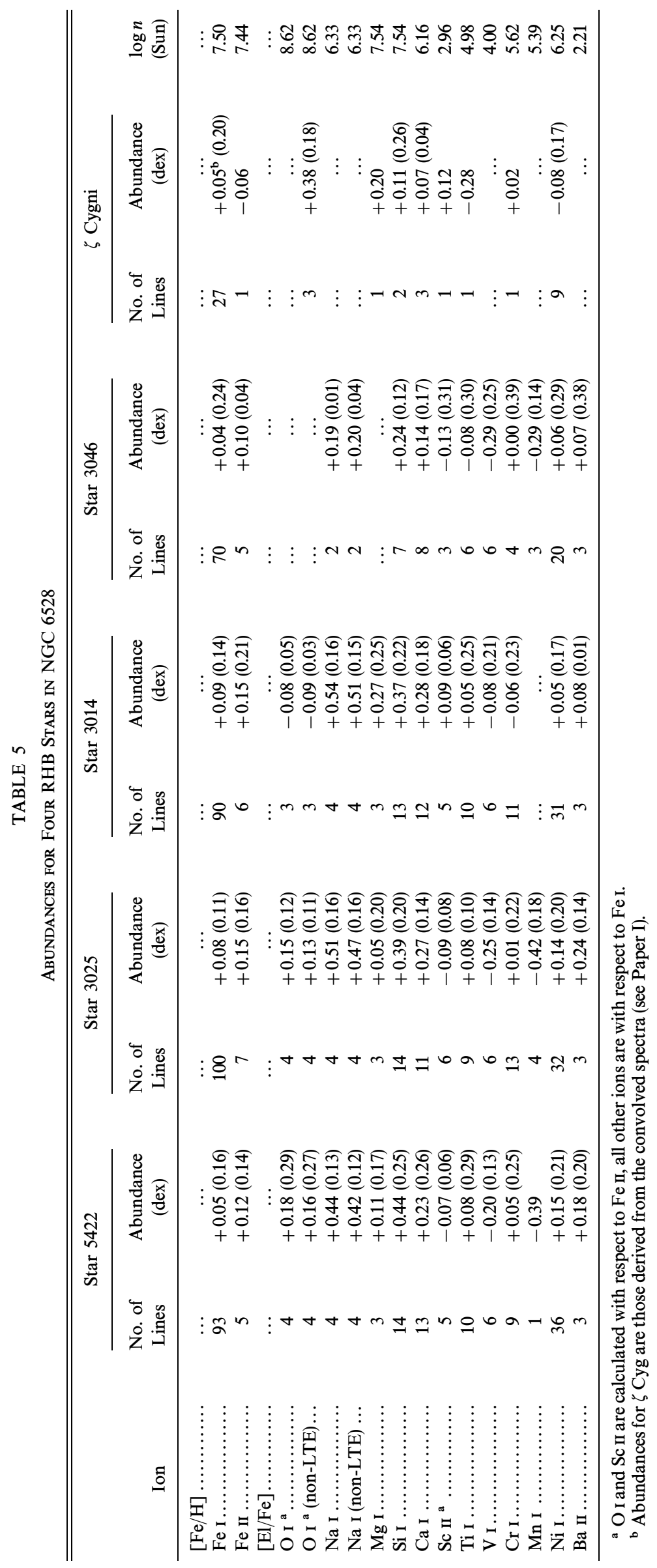




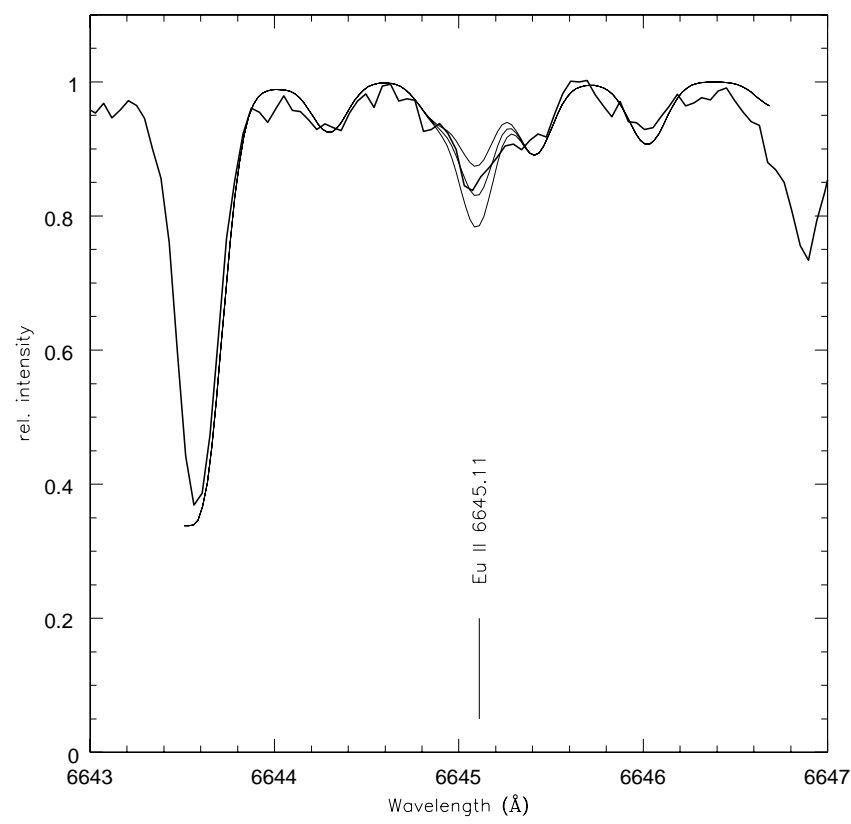

FIG. 5.-Comparison between the observed spectrum in the region of the Eu II line at $6645.11 \AA$ (average of all stars in NGC 6528 ; thick line) and synthetic spectra computed for $[\mathrm{Eu} / \mathrm{Fe}]=0.0 .0 .25,0.50$ (thin lines). From this comparison, evidence for a mild $([\mathrm{Eu} / \mathrm{Fe}] \simeq 0.2)$ overabundance of $\mathrm{Eu}$ is found.

reminded by the second referee, RHB stars have already experienced the phase along the RGB where $\mathrm{C}$ is usually depleted and $\mathrm{N}$ enhanced, and their sum is constant.) The synthetic spectra were then convolved with a Gaussian with a FWHM of $0.12 \AA$, in order to match the resolution of our spectra. We examined the profiles and flagged all lines

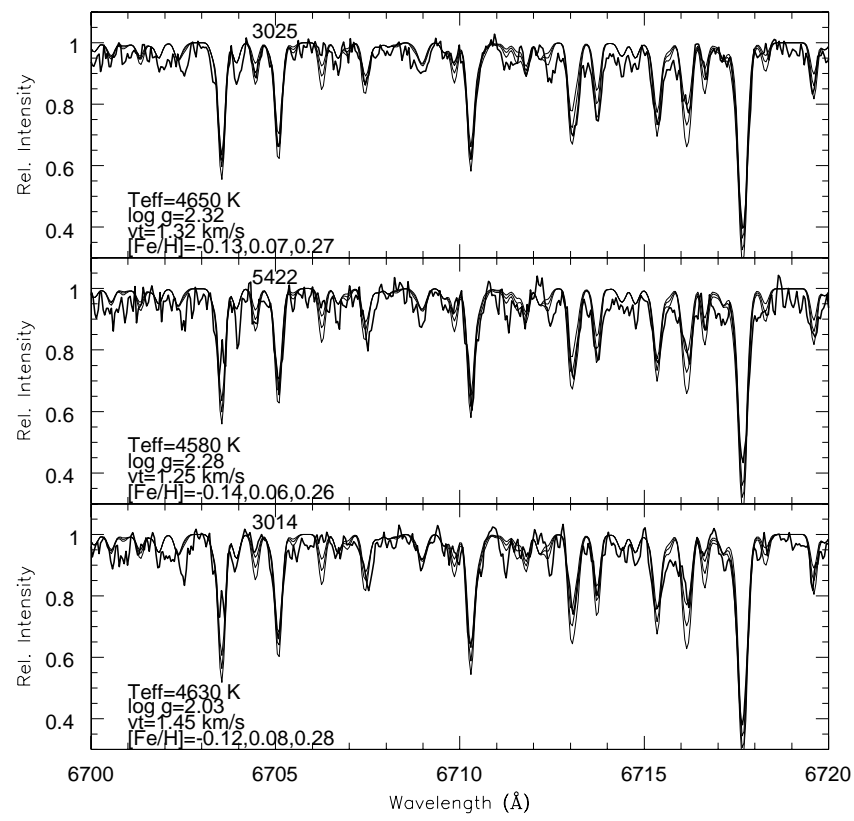

FIG. 6.-Spectral synthesis of the region around the Li doublet for the three RHB stars of NGC 6528, with spectra taken in the run of 2000 June. Thick lines are the observed spectra, while the thin lines are predictions for model atmospheres, with the parameters indicated in each box for abundances of $[\mathrm{Fe} / \mathrm{H}]$ listed at the bottom of each panel. Wavelengths have been shifted into the rest frame. whose profiles are in some way distorted by blends; we then measured the EWs of the lines on these synthetic spectra using a Gaussian fitting routine (all parameters left free; EWs obtained by this procedure are much more affected by the presence of blends than those obtained using the procedure we applied for the program stars).

These EWs were then compared with those obtained from a spectral synthesis where the line list only consisted of the line under scrutiny (this time using a simple integration over the profile). Next, we flagged all lines where the two EWs differ by more than $2 \mathrm{~mA}$ (note that the weakest $\mathrm{Fe}$ I lines have EW > $40 \mathrm{~m} \AA$ ). Finally, we adopted as very clean lines those with no appreciable distortion in the profiles and for which the EWs are not changed by more than $2 \mathrm{~m} \AA$ by blends. We find a total of 53 very clean Fe I lines; such lines are marked with an asterisk in Table 2. Average abundances obtained from this subset of lines are within 0.020 dex of those derived from the original sample, which in addition included lines of somewhat poorer quality. Furthermore, the rms scatter of individual values are not modified in a significant way.

This exercise suggests that (1) the procedure used to measure EWs (i.e., adopting an average relation between EWs and FWHM to constrain the FWHM) allows us to derive reliable EWs even for moderately blended lines, as in the spectrum of star 3046; and (2) that the largest source of errors in the EWs is the location of the continuum level rather than the presence of blends.

Moreover, following the same procedure as in Paper I, we checked our overall $[\mathrm{Fe} / \mathrm{H}]$ values by comparing our spectra with syntheses of a spectral region around the $\mathrm{Li}$ doublet, which includes several weak or medium-strength $\mathrm{Fe}$ lines. These comparisons are shown in Figure 6, only for the three stars having new, high-quality spectra from the run of 2000. The remaining star, 3046, was tied on the EW system defined from the three others as explained above.

The comparisons between the spectral syntheses and the observed spectra for the RHB stars in NGC 6528 shown in Figure 6 support the abundances found by the analysis of the equivalent widths. Lines computed with $[\mathrm{Fe} / \mathrm{H}]=$ -0.13 and $[\mathrm{Fe} / \mathrm{H}]=+0.27$ are clearly too shallow and too strong with respect to the observations. Figure 6 also shows that no lithium is detectable in all our program stars. The synthetic spectra in Figure 6 are computed with $\log n(\mathrm{Li})=-2^{6}$; however, since the $\mathrm{Li}$ line is blended with a Fe line, the upper limit determined from our spectra is $\log n(\mathrm{Li})<0$.

We also note that if we only use $\mathrm{Fe}$ I lines with $\log g f-\theta \chi \leq-7.2$ (where $\theta=5040 / T$ ), which corresponds to the weakest lines that could be detected in the spectra taken in the 2000 June run, we obtain $[\mathrm{Fe} / \mathrm{H}]=+0.08 \mathrm{dex}$ using 14 lines for star $3025,[\mathrm{Fe} / \mathrm{H}]=+0.09$ for star 5422 using 11 lines, and $[\mathrm{Fe} / \mathrm{H}]=+0.06$ for 12 lines in star 3014, these values are indistinguishable from those obtained with the full set of $\mathrm{Fe}$ I lines. Abundances from such weak lines are almost independent of the choice of microturbulent velocity. Although expected by the manner in which $v_{t}$ is set, the close agreement in the derived $\mathrm{Fe}$ abundance between

${ }^{6}$ For lithium abundances, we used the usual notation: $\log n(\mathrm{~A})$ is the abundance (by number) of the element $\mathrm{A}$ in the usual scale, where $\log n(\mathrm{H})=12$; for all other elements, we use instead the notation $[\mathrm{A} / \mathrm{H}]$, which is the logarithmic ratio of the abundances of elements $A$ and $H$ in the star minus the same quantity in the Sun. 
the set of weak lines and the full set of $\mathrm{Fe}$ I lines is reassuring.

\section{DISCUSSION OF RESULTS}

The present results are summarized in Table 6, where we also list the mean abundances for NGC 6553 (both from Paper I and from B99) and for the 11 giants in Baade's window studied by McWilliam \& Rich $(1994)^{7}$ in order to provide a deeper insight into our findings. For NGC 6528 and our analysis of NGC 6553, if there was only one line per star for a given ion, the value is given in parentheses.

\subsection{Comparisons of $\mathrm{Fe}$ Abundance}

The most meaningful and immediate comparison is with our results for NGC 6553 from Paper I, since the analysis technique and data set are very consistent and homogeneous. However, both the quality of the observational material and the approach to the EW measurements present some differences.

Therefore, the first test we performed was a direct comparison of spectra of stars in the two clusters. RHB stars are objects in a well-defined evolutionary phase. Hence, we can expect their stellar structure and parameters to be very similar at similar metallicities.

This is evident from Figure 7, where the spectrum of star 71 in NGC 6553 (from Paper I) is compared with that of star 3025 of NGC 6528 (from the present work) in the spectral region 6700-6740 $\mathrm{A}$. For a meaningful comparison, the spectra have been degraded to the same resolution. This figure shows that the spectra of the two stars are actually very similar, apart from some small differences in the positioning of the continuum. We conclude that the two stars can hardly be considered different or even distinguishable on the basis of their spectra.

\footnotetext{
${ }^{7}$ Rich \& McWilliam (2000) present a preliminary report suggesting that from their higher S/N HIRES spectra, they estimate that their previously published $\mathrm{Fe}$ abundances for galactic bulge giants need to be revised upward by $0.1-0.2$ dex for stars more metal-rich than the Sun.
}
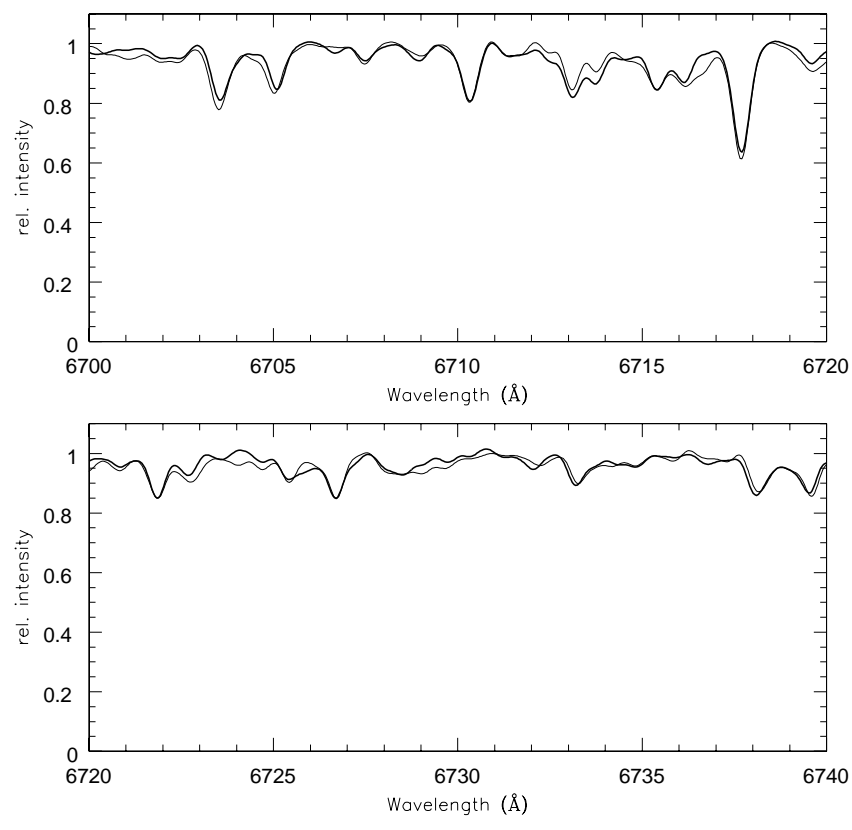

FIG. 7.-Comparison of spectra of two RHB stars in NGC 6553 (star 71, see Paper I; thin line) and in NGC 6528 (star 3025 from present work; thick line). Spectra have been degraded to the same resolution.

This idea is strongly supported by Figure 8 , where average EWs from stars of NGC 6553 (Paper I) and NGC 6528 (present study) are compared. In order to make the comparison more robust and less dependent on details of the continuum tracing, we average only EWs of lines measured in at least three stars out of five in NGC 6553 and in at least two stars out of the three with the better quality spectra in NGC 6528. Again, this comparison is quite good and EWs are quite similar for a hypothetical average RHB star in the two clusters.

If the observed spectra are the same and the equivalent widths are very similar, then the difference of about 0.2 dex in the average overall metallicity of these two globular clus-

TABLE 6

MeAn Abundances for NGC 6528 and Comparison with Results For NGC 6553 AND FOR BaAde's Window

\begin{tabular}{|c|c|c|c|c|c|c|c|c|}
\hline Ion & $\begin{array}{l}\text { NGC } 6528 \\
\quad(\text { dex })\end{array}$ & $\begin{array}{c}\sigma \\
(\operatorname{dex})\end{array}$ & $\begin{array}{c}\text { NGC } 6553 \text { Paper I } \\
\text { (dex) }\end{array}$ & $\begin{array}{c}\sigma \\
(\mathrm{dex})\end{array}$ & $\begin{array}{c}\text { NGC } 6553 \text { Barbuy et al. } \\
\text { (dex) }\end{array}$ & $\begin{array}{c}\sigma \\
(\operatorname{dex})\end{array}$ & $\begin{array}{l}\text { Mean BWa } \\
\quad(\text { dex })\end{array}$ & $\begin{array}{c}\sigma \\
(\operatorname{dex})\end{array}$ \\
\hline$[\mathrm{Fe} / \mathrm{H}] \ldots \ldots$ & $\ldots$ & $\ldots$ & $\ldots$ & $\ldots$ & $\ldots$ & $\ldots$ & $\ldots$ & $\ldots$ \\
\hline Fe $1 \ldots \ldots \ldots$ & +0.07 & 0.02 & -0.16 & 0.08 & -0.55 & 0.20 & -0.33 & $\ldots$ \\
\hline Fe II .......... & +0.13 & 0.02 & -0.18 & 0.10 & $\ldots$ & $\ldots$ & $\ldots$ & $\ldots$ \\
\hline$[\mathrm{El} / \mathrm{Fe}] \ldots \ldots$ & $\ldots$ & $\ldots$ & $\ldots$ & $\ldots$ & $\ldots$ & $\ldots$ & $\ldots$ & $\ldots$ \\
\hline $\mathrm{O}_{\mathrm{I}} \ldots \ldots \ldots$ & +0.07 & 0.11 & +0.50 & 0.13 & $\ldots$ & $\ldots$ & +0.03 & 0.18 \\
\hline $\mathrm{Na}$ I $\ldots . . . .$. & +0.40 & 0.12 & $\ldots$ & $\ldots$ & +0.65 & 0.05 & +0.21 & 0.37 \\
\hline $\operatorname{Mg}{ }_{1} \ldots \ldots \ldots$ & +0.14 & 0.09 & $(+0.41)^{\mathbf{b}}$ & $(0.10)$ & +0.33 & 0.13 & +0.35 & 0.14 \\
\hline Si I $\ldots . . . \ldots$. & +0.36 & 0.07 & +0.14 & 0.18 & +0.35 & 0.05 & +0.18 & 0.24 \\
\hline 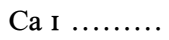 & +0.23 & 0.06 & +0.26 & 0.09 & +0.32 & 0.12 & +0.14 & 0.17 \\
\hline Sc II ......... & -0.05 & 0.10 & $(-0.12)$ & $(0.18)$ & $\ldots$ & $\ldots$ & +0.29 & 0.20 \\
\hline Ti I $\ldots . . . \ldots$ & +0.03 & 0.07 & +0.19 & 0.06 & +0.51 & 0.09 & +0.34 & 0.10 \\
\hline$V_{i} \ldots \ldots \ldots$ & -0.20 & 0.09 & $\ldots$ & $\ldots$ & $\ldots$ & $\ldots$ & +0.06 & 0.19 \\
\hline Cr I $\ldots \ldots \ldots$ & 0.00 & 0.04 & +0.04 & 0.09 & $\ldots$ & $\ldots$ & -0.04 & 0.19 \\
\hline Mn I $\ldots . . .$. & -0.37 & 0.07 & $\ldots$ & $\ldots$ & $\ldots$ & $\ldots$ & $\ldots$ & $\ldots$ \\
\hline Ni $1 \ldots . . . \ldots$. & +0.10 & 0.05 & +0.01 & 0.07 & $\ldots$ & $\ldots$ & -0.04 & 0.08 \\
\hline Ba II ......... & +0.14 & 0.07 & $\ldots$ & $\ldots$ & -0.10 & 0.30 & +0.20 & 0.28 \\
\hline
\end{tabular}

a Abundances for Baade's window are from the 11 giants studied by McWilliam \& Rich 1994.

b Parentheses indicate ions for which a maximum of one line has been observed per star. 


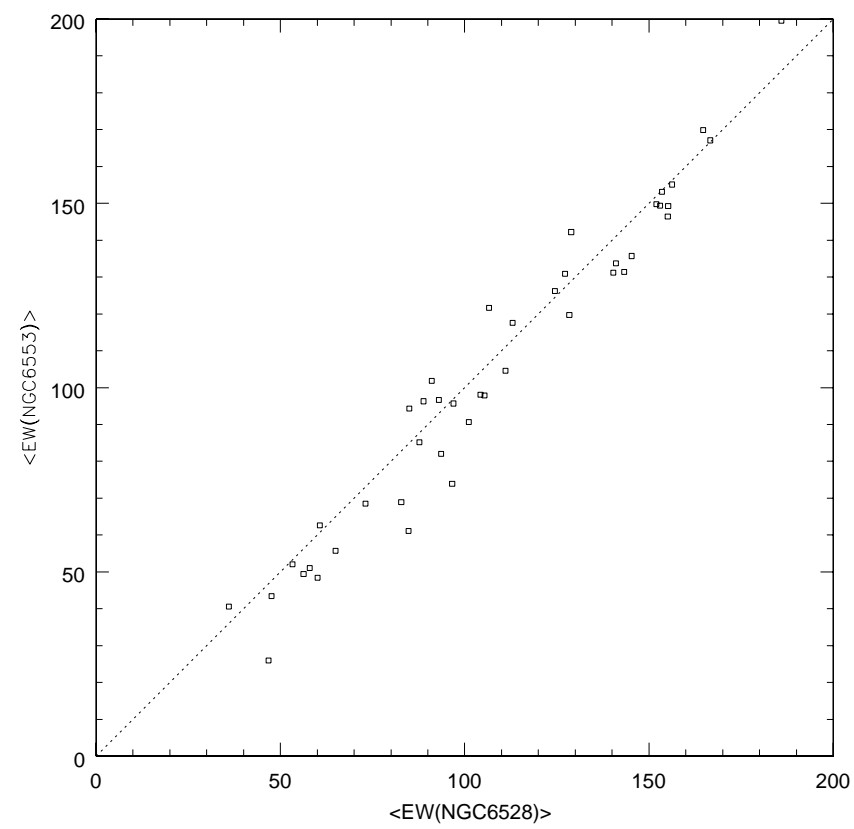

FIG. 8.-Comparison of average EWs for RHB stars in NGC 6553 and 6528. The average is from the five stars studied in Paper I and is shown when a line is measured in at least three stars, for NGC 6553; for NGC 6528 , the average was done using the three stars with spectra taken in the 2000 run and only if a line was measured in at least two stars.

ters $([\mathrm{Fe} / \mathrm{H}]=-0.16$ for $\mathrm{NGC} 6553$ and $[\mathrm{Fe} / \mathrm{H}]=+0.07$ dex in NGC 6528) must arise from the different atmospheric parameters adopted in the two analysis. In fact, both here and in Paper I, the atmospheric parameters adopted were derived directly from spectra, i.e., from EWs, that in turn were measured on spectra of different quality (better for the NGC 6528 stars observed in the run of 2000 June) and using different methods.

To verify this, we use Table 4 to estimate the changes in $[\mathrm{Fe} / \mathrm{H}]$ due to differences between the mean atmospheric parameters used for RHB stars in NGC 6553 (namely, 4727, $2.3,-0.13$, and 1.82 for temperature, gravity, model abundance, and microturbulent velocity, respectively) and the mean set used for NGC 6528 (namely, 4620, 2.21, + 0.07, and 1.34). The resulting difference in $[\mathrm{Fe} / \mathrm{H}]$ is -0.24 , in the sense that an average star in NGC 6553 should be more metal-poor than the average one in NGC 6528. The small difference in adopted microturbulence of only $0.5 \mathrm{~km} \mathrm{~s}^{-1}$ gives rise to most of the abundance difference.

Another experiment seems to confirm this finding. We used the set of average EWs for NGC 6528 and repeated the analysis. We derive the values $4640,2.28,0.08$, and 1.37 for $T_{\text {eff }}, \log g,[\mathrm{~A} / \mathrm{H}]$, and $v_{t}$, and obtain $[\mathrm{Fe} / \mathrm{H}]=+0.08$ using the procedures discussed in $\S 3.1$ zeroing trends of abundance from single lines with $\chi$ and expected line strengths. With these values, we also obtained a very good ionization equilibrium.

If we now repeat the abundance analysis applying this set of atmospheric parameters to the average EWs of NGC 6553 , we obtain a solution where the slopes of linear regressions of abundances versus $\chi$ and of expected line strengths are well within the $1 \sigma \mathrm{rms}$ uncertainty. The resulting abundance for NGC 6553 is then $[\mathrm{Fe} / \mathrm{H}]=+0.04$, i.e., we can conclude that an acceptable value for the overall metallicity of this cluster, as estimated from the $\mathrm{Fe}$ I abundance, is only 0.04 dex lower than that of NGC 6528.
Finally, Figure 9 shows the parameter space $v_{t}, T_{\text {eff }}$ over the regime expected for RHB stars. Using the average EWs, we show the contours that give errors in slopes of linear regressions of abundances versus $\chi$ and of expected line strengths within the $1 \sigma \mathrm{rms}$ uncertainty. The loci for both NGC 6528 (solid line) and for NGC 6553 (dotted line) are elongated. This is simply another, graphic representation of the coupling that exists between $v_{t}$ and $T_{\text {eff }}$ for the cool, metal-rich stars discussed above (\$ 3.1).

The higher quality spectra taken in the second run for NGC 6528 do alleviate this phenomenon, permitting highprecision measurements of EWs of weak lines also with high excitation. This, in turn, allows us to better constrain the region of this parameter space, within which solutions of the abundance analysis are acceptable.

These tests support the conclusion that within the error bars, the overall metal abundance of the two clusters expressed in terms of $[\mathrm{Fe} / \mathrm{H}]$ is virtually the same. This is a firm result, since the number of measured $\mathrm{Fe}$ lines is large. Taking into account the overall uncertainty, we can be quite confident that NGC 6528 is a close "twin" of NGC 6553 , as far as the overall metal abundance is concerned. Both these bulge clusters have a metallicity slightly larger than solar. Extremely high abundances for NGC 6528, as well as NGC 6553, can be safely ruled out, as confirmed also by their normal integrated colors (see Fig. 8 in Feltzig \& Gilmore 2000).

To be conservative, we will adopt hereafter a value of metallicity for NGC 6553 that is the mean of that obtained in Paper I and the one obtained with atmospheric parameters equal to those of NGC 6528. As the error bar, we will adopt one giving these two values as extremes of our confidence range. Hence, we will adopt for NGC 6553 a value of $[\mathrm{Fe} / \mathrm{H}]=-0.06 \pm 0.15$ dex.

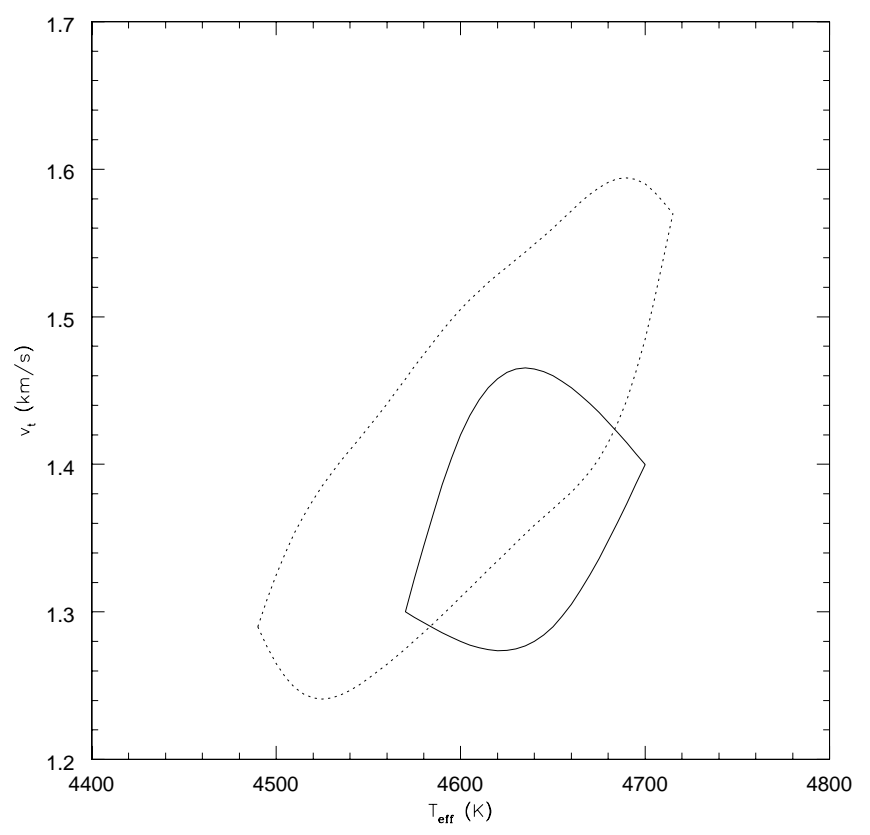

FIG. 9.-Parameter space $v_{t}, T_{\text {eff }}$ in the range for RHB stars. The closed lines define the loci where solutions can be obtained with errors in slopes of linear regressions of abundances vs. $\chi$ and of expected line strengths within the $1 \sigma \mathrm{rms}$ uncertainty. The dotted line refers to solutions for NGC 6553, while the solid one is for solutions valid for NGC 6528, for which higher quality spectra and EWs are available (see text). 


\subsection{Comparison with NGC 6553 for Other Elements}

The pattern described by the element ratios appears to be rather similar for the two clusters. The average abundances are generally in good agreement, apart from the $[\mathrm{Mg} / \mathrm{Fe}]$ ratio. However, as discussed in $\S 2.2$, with the present instrumental setup, we had no access to the $\mathrm{Mg}$ line at $8717.8 \AA$, the clean line used in the analysis of RHB stars in NGC 6553. Instead, we had to use two lines around $5600 \AA$, which are located in a more crowded region and are saturated, hence not optimal for a good abundance derivation. Even if the abundance from the line at $7657 \AA$, which we measured for stars observed in the 2000 run, generally confirms results from the other lines, at present and until further confirmation, we cannot be very confident that the low $\mathrm{Mg}$ abundance found for NGC 6528 is real.

The overabundance of $\mathrm{Si}$ and of $\mathrm{Ca}$ seems to be well established in NGC 6528. Since, however, O abundances (discussed later) and Ti abundances seem to be more likely solar, it is not clear that NGC 6528 presents the classical overabundance of the $\alpha$-elements typical of more metalpoor clusters and field stars.

On the other hand, $\mathrm{Mn}$ is rather underabundant in NGC 6528 , and this result is a signature of a small contribution to nucleosynthesis by SNe Ia, suggesting that the $\alpha$-elements overabundance might be the typical fossil record of a nucleosynthesis history heavily dominated by massive stars. Some support to this scenario could be given by the mild overabundance of $\mathrm{Eu}$ found from the combined spectrum of the RHB stars in NGC 6528, since Eu is known to be an $n$-capture element predominantly produced by $r$-processes. Note, however, that the ratio $[\mathrm{Ba} / \mathrm{Eu}]$ is almost solar, suggesting that perhaps we are also seeing the contribution to nucleosynthesis from intermediate-mass stars.

A comparison of our results for NGC 6553 with those of B99 was given in $\S 7.1$ of Paper I. Here we note only that the choice of solar and stellar atmosphere models adopted in B99 (hence, the solar abundances) results in $\mathrm{Fe}$ abundances that are $0.1-0.15$ dex lower than those of the present scale. This by itself decreases the differences in the element ratios presented in Table 6. In other words, had the comparison for NGC 6553 been made, instead, in terms of [element/H], the pattern of many elements would have been more similar when our analysis of Paper I was compared with that of B99.

\section{3. $\mathrm{O}$ and $\mathrm{Na}$ Abundances}

The slightly different instrumental configuration of HIRES adopted for the observations of the RHB stars in NGC 6528 allowed the use of the weak Na doublet at 6154, $6160 \AA$, as well as the infrared permitted O I triplet, and, for three of the four stars, the forbidden $6300 \AA$ O I line as well.

To take into account departures from the adopted assumption of LTE, appropriate corrections were applied to $\mathrm{O}$ and $\mathrm{Na}$ abundances following the prescriptions of Gratton et al. (1999). Non-LTE abundances from the line analysis are also listed in Table 5. Note, however, that the corrections are rather small and do not exceed a few hundredths of a dex in all cases.

We tested the abundances we derived for $\mathrm{Na}$ and $\mathrm{O}$ using synthetic spectra of the $6154,6160 \AA \mathrm{Na}$ I doublet and of the [O I] line at $6300 \AA$. The spectral syntheses were computed assuming LTE. Comparisons with spectral syntheses for these two spectral regions are shown in Figures 10 and 11, respectively.

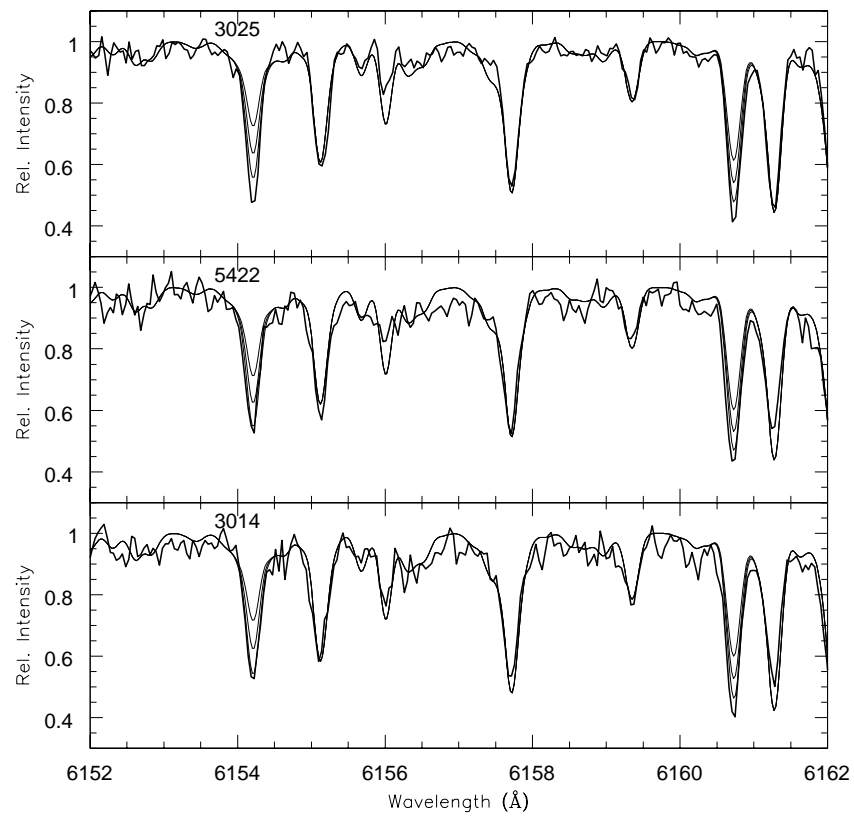

Fig. 10.- Spectrum synthesis of the region of $\mathrm{Na}$ doublet at 6154 and $6160 \AA$ for the RHB stars analyzed in NGC 6528 (only for stars observed in 2000). The thick lines are the observed spectra, while the thin lines are predictions for abundances of $[\mathrm{Na} / \mathrm{Fe}]=0.0,0.2$, and 0.4 dex.

From the comparisons in Figure 10, we found that $\mathrm{Na}$ is overabundant by about +0.35 to +0.5 dex in stars 3025 , 5422 , and 3014 (respectively, we estimated $[\mathrm{Na} / \mathrm{Fe}]=+0.5$, +0.35 , and $+0.4 \mathrm{dex})$. These results are consistent with that deduced from the EWs.

The comparison between observed and synthesized spectra for the region, including the [O I] line at $6300.3 \AA$, is

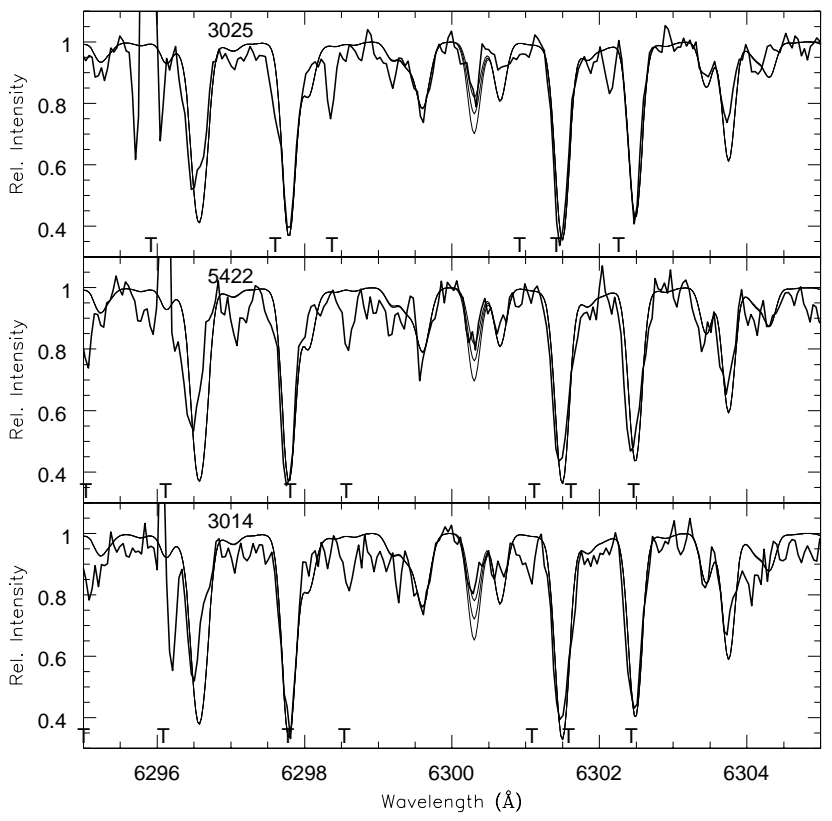

FIG. 11.-Spectrum synthesis of the region of the [O I] forbidden line at $6300 \AA$ of stars in NGC 6528 observed in the 2000 run. Thick lines are the original observed spectra, while the thin lines denote predictions for abundances of $[\mathrm{O} / \mathrm{Fe}]=-0.07,0.13$, and 0.33 dex. The spectra were computed assuming $[\mathrm{C} / \mathrm{Fe}]=-0.45,-0.30$, and -0.18 , and $[\mathrm{N} / \mathrm{Fe}]=0$; these choices reproduce the strength of the $\mathrm{CN}$ lines. Here T's mark telluric features. 
more difficult for three reasons. First, there are several telluric lines in this region whose locations are marked with a " $T$ " in Figure 11. Because of the high $v_{r}$ of NGC 6528, the wavelength of the $\left[\mathrm{O}_{\mathrm{I}}\right]$ line in the observed spectra of NGC 6528 is free of contamination by telluric lines; they should not affect our results. Second, as mentioned in $\S 3.1$, O abundances are quite sensitive to uncertainties in the atmospheric parameters. Third, there is some coupling between $\mathrm{C}$ and $\mathrm{O}$ abundances due to the formation of $\mathrm{CO}$ in the atmospheres of these cool, metal-rich $\mathrm{K}$ giants. We estimated that derived $\mathrm{O}$ abundances increase by 0.06 dex if the $\mathrm{C}$ abundances are raised by 0.2 dex. Unfortunately, we could not determine $\mathrm{C}$ abundances from our spectra, since EWs of $\mathrm{C}$ I lines measured in the red are not reliable; we can only fix the overall strength of $\mathrm{CN}$ lines. We adequately matched $\mathrm{CN}$ lines by assuming $[\mathrm{C} / \mathrm{Fe}]=-0.45$ and $[\mathrm{N} / \mathrm{Fe}]=0$ when $[\mathrm{O} / \mathrm{Fe}]=-0.07,[\mathrm{C} / \mathrm{Fe}]=-0.3$ and $[\mathrm{N} / \mathrm{Fe}]=0$ when $[\mathrm{O} / \mathrm{Fe}]=0.13$, and $[\mathrm{C} / \mathrm{Fe}]=-0.18$ and $[\mathrm{N} / \mathrm{Fe}]=0$ when $[\mathrm{O} / \mathrm{Fe}]=+0.33$. These are the values adopted in the syntheses shown in Figure 11.

Figure 11 shows that with the adopted $\mathrm{C}$ and $\mathrm{N}$ abundances, a good fit of the $[\mathrm{O} \mathrm{I}]$ line is obtained for $[\mathrm{O} /$ $\mathrm{Fe}] \sim 0$, in reasonable agreement with the results given by the analysis of the equivalent widths (where also other $\mathrm{O}$ lines are taken into account). An $\mathrm{O}$ excess of 0.3 dex seems excluded, unless the $\mathrm{C}$ abundance is not much larger than solar, which seems unlikely. We conclude that the program stars in NGC 6528 have solar oxygen abundances, but a distinct excess of $\mathrm{Na}([\mathrm{Na} / \mathrm{Fe}] \sim 0.4)$.

\subsection{Metal Abundances: Calibration of Other Indices}

Our need, after the completion of our analysis of NGC 6553 of Paper I, to have an analysis for stars in at least one more metal-rich cluster is apparent if one examines Figure 12 , where the metallicities obtained from high-resolution spectra by CG97, plus those for NGC 6553 and NGC 6528 (this study) are plotted against the parameter $W(\mathrm{Ca}$ II) determined by Rutledge et al. (1997a, 1997b) from the measured strength of the infrared Ca triplet in individual globular cluster giants.

For reasons explained in $\S 4.1$, we believe that the correct $[\mathrm{Fe} / \mathrm{H}]$ values to adopt for these two clusters are +0.07 and -0.06 dex for NGC 6528 and 6553 , respectively.

When NGC 6528, which has the largest value of any galactic globular cluster observed to date on the Rutledge et al. ranking scale $[W(\mathrm{Ca} \mathrm{II})=5.41]$, is added, the conclusion proposed in Paper I is still firmly supported. Specifically,

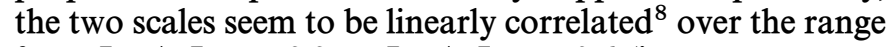
from $[\mathrm{Fe} / \mathrm{H}]=-2.2$ to $[\mathrm{Fe} / \mathrm{H}]=-0.6$ (i.e., up to not so extremely metal-rich clusters). At higher metallicities, however, where the $\mathrm{Ca}$ II index is known to lose its sensitivity to metal abundance (see Armandroff \& Da Costa 1991), the linear correlation seems to break down. Note that clusters like Pal 12 or Rup 106, known to have anomalously low $[\alpha / \mathrm{Fe}]$ ratios, are not included in the sample.

With our new results, we can now better assess the relationship between the two scales: in fact, NGC 6528 falls very near NGC 6553 in the $[\mathrm{Fe} / \mathrm{H}]-W(\mathrm{Ca}$ II) plane and strongly constrains the position of metal-rich globular clusters for the metallicity calibration in terms of this low-

\footnotetext{
${ }^{8}$ Note that in Figure 12, a dashed line indicates the linear fit that one would obtain using all clusters just for purpose of comparison.
}

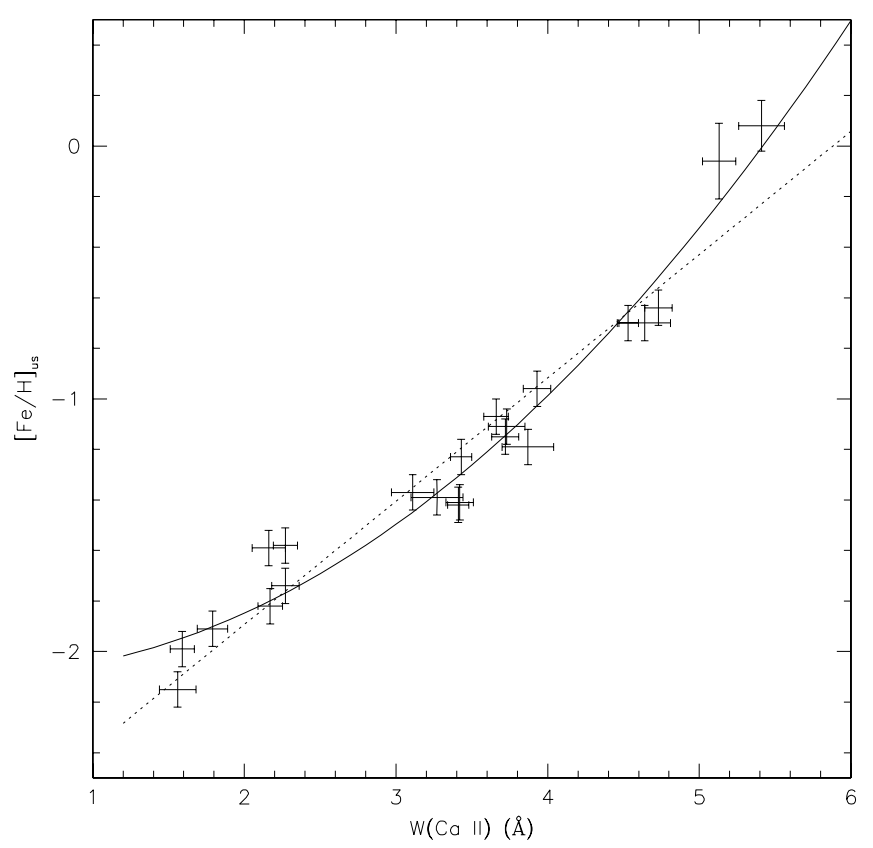

FIG. 12.-Parameter $W(\mathrm{Ca}$ II) defined by Rutledge, Hesser, \& Stetson (1997a) shown as a function of abundance on the scale of Carretta \& Gratton (1997) for galactic globular clusters with high-dispersion analyses, as updated by the analysis by Cohen et al. (1999) for NGC 6553 (but see text, $\S 4.3$ ) and the present study for NGC 6528. The dashed line is a linear fit shown for comparison purposes. The solid line is the transformation adopted (see text).

resolution indicator. This is not unexpected, since in $\S 4.1$ we showed how RHB stars of these two clusters are similar. We find that to bring the values from Rutledge et al. onto a homogeneous metallicity scale that is based completely on high-dispersion spectroscopy, one has to adopt a secondorder polynomial relation

$$
[\mathrm{Fe} / \mathrm{H}]_{\mathrm{us}}=-2.08-0.04 W(\mathrm{Ca} \mathrm{II})+0.078 W^{2}(\mathrm{Ca} \mathrm{II}) \text {, }
$$

with the correlation coefficient $r=0.98$ and $\sigma=0.12 \mathrm{dex}$ for 22 clusters. The second-order term is significant at a confidence level exceeding $95 \%$; inclusion of this term yields $\chi^{2}=25.8$ for the sample of 22 globular clusters in common. This is a reduction in $\chi^{2}$ of a factor of 1.6 compared with a linear fit. Note that similar results are obtained even if we omit NGC 6553 entirely, so that the larger error bar of the revised $[\mathrm{Fe} / \mathrm{H}]$ value for this cluster does not affect much our calibration.

This relation, shown in Figure 12, allows us to derive directly from the $\mathrm{Ca}$ index of a globular cluster its metallicity on the Carretta \& Gratton scale, as extended here and in Paper I. The range of application should be restricted to that of calibrating clusters, namely from $W(\mathrm{Ca}$ II) $\sim 1.5$ (NGC 7078 and 4590) to $W$ (Ca II) 5.4 (NGC 6528). Moreover, using data from the compilation of Carney (1996), we checked that the differences between the $W(\mathrm{Ca}$ II) values as observed by Rutledge et al. and the $W(\mathrm{Ca}$ II) values predicted from our equation (2) are constant as a function of $[\mathrm{Ca} / \mathrm{Fe}]$. We can then conclude that, since the $[\mathrm{Ca} / \mathrm{Fe}]$ ratio holds rather constant for all clusters in the sample, we are actually calibrating an index that is tied to the temperatures and luminosities of stars along the RGB, which in turn depend on the cluster metal abundance.

Adding a second, well-analyzed, high-metallicity cluster is also very useful in intercomparing the CG97 scale and the 
widely used scale of Zinn \& West (1984; based on integrated light indices). We are now able to derive a relation to bring their values on the new scale entirely based on highdispersion analyses without using any uncertain extrapolations. As in CG97, we prefer to use final metallicities directly from Zinn \& West (1984), with the update of Armandroff \& Zinn (1988; collectively ZW) because of the variety of indicators used in the analysis of Zinn \& West (1984).

When NGC 6528 and 6553 are added to the other 24 calibrating clusters, the quadratic relation previously found by CG97 is no longer the most appropriate, as one can see in Figure 13, where mean metallicities from the CG97 scale, as augmented here, are compared with metallicities on the ZW scale.

In this case, a cubic polynomial is the best relation to transform the ZW scale to the new high-dispersion spectroscopic scale:

$$
\begin{aligned}
{[\mathrm{Fe} / \mathrm{H}]_{\mathrm{CG}}=+0.61+3.04[\mathrm{Fe} / \mathrm{H}]_{\mathrm{ZW}} } & +1.981[\mathrm{Fe} / \mathrm{H}]_{\mathrm{ZW}}^{2} \\
+ & 0.532[\mathrm{Fe} / \mathrm{H}]_{\mathrm{ZW}}^{3},
\end{aligned}
$$

with $\sigma=0.10$ dex and a correlation coefficient $r=0.99$ for 26 clusters.

When considering the adopted error bar of 0.15 dex for the revised value of $[\mathrm{Fe} / \mathrm{H}]$ of NGC 6553 , the second- and third-order terms are significant at the $99 \%$ confidence level.

The value of $\chi^{2}$ decreases by more than a factor of 2 in going from a linear fit to a third-order fit, e.g., from 392 to 144 for the comparison of our abundances with those of Zinn \& West (1984) for the 26 clusters (25 without NGC $6553)$ in the sample with high-dispersion abundance analyses.

The present work extends the range of application of this transformation by about 0.3 dex toward high metallicity with respect to equation (7) in CG97. Using the above relation now allows one to transform the $\mathrm{ZW}$ metallicities onto

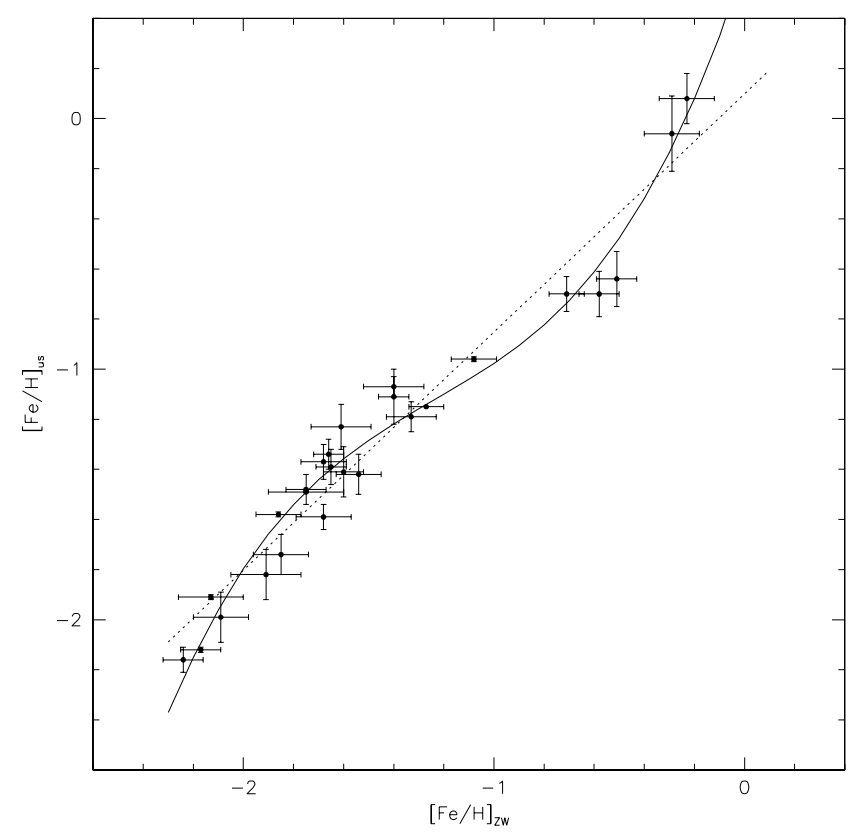

FIG. 13.-New calibration of the metallicity scale by Zinn \& West (1984) vs. metal abundances from high-dispersion spectroscopy. The dashed and solid lines have the same meaning as in Fig. 12. our high-dispersion scale in the range $-2.24<[\mathrm{Fe} / \mathrm{H}]_{\mathrm{Zw}}$ $<+0.12$.

\section{CONCLUSIONS}

We present for the first time an abundance analysis for stars in the metal-rich bulge cluster NGC 6528 based on high-resolution spectra of $\mathrm{S} / \mathrm{N}$ high enough to allow a reliable fine abundance analysis.

We observed four red horizontal-branch stars, from which we found a mean $[\mathrm{Fe} / \mathrm{H}]=+0.07 \pm 0.01$ dex, with a star-to-star scatter of $\sigma=0.02$ (not including systematic effects).

This metallicity is slightly larger than the mean abundance in the galactic bulge found by McWilliam \& Rich (1994) and that found in a previous paper for NGC 6553. However, we found that the spectra of stars in NGC 6553 and 6528 and the EWs we measured on them are very similar to each other. The slightly different metal abundance is the result primarily of adoption of slightly lower microturbulent velocity in the analysis of NGC 6528 stars and secondarily of the adoption of slightly lower temperatures for the NGC 6528 stars.

Note that the present analysis is based on higher quality spectra, so that the atmospheric parameters used here for NGC 6528 are more firmly established than those used in Paper I for NGC 6553.

If the same atmospheric parameters are adopted for both samples, nearly equal metal abundances are derived for the two clusters. We then propose to revise upward the metal abundance of NGC 6553 to $[\mathrm{Fe} / \mathrm{H}]=-0.06 \pm 0.15$, where the error bar encompasses both the original abundance for NGC 6553 we derived in Paper I and the value we obtained assuming in the analysis atmospheric parameters identical to those we used for stars in NGC 6528.

These results imply that metal-rich globular clusters may reach or even exceed the mean abundance of the galactic bulge found by McWilliam \& Rich but are found to fall within the spread of their distribution.

The relative abundance for the best determined $\alpha$-process elements $(\mathrm{Ca})$ indicates an excess of $\alpha$-process elements of about a factor of 2 and a global pattern of abundances similar to that of NGC 6553 and the bulge field stars. Moreover, Mn in NGC 6528 clearly shows an underabundance typical of a fairly small contribution to nucleosynthesis by $\mathrm{SNe}$ Ia. When coupled with the overabundances of $\mathrm{Si}$ and $\mathrm{Ca}$, our results strongly suggest that these bulge globular clusters seem to have experienced a history of chemical enrichment essentially identical to that typical of bulge field stars, probably under conditions of enrichment by Type II supernovae at early epochs.

We found a solar $\mathrm{O}$ abundance, while there is a clear excess of $\mathrm{Na}([\mathrm{Na} / \mathrm{Fe}] \sim 0.4)$. However the sample is not large enough to understand whether this abundance pattern is characteristics of all stars in this cluster or rather stems from peculiarities of the program stars or their evolutionary state.

Our new data for NGC 6553 (Paper I) and NGC 6528 allow us to recalibrate the widely used metallicity scales of Zinn \& West (1984) and of Rutledge et al. (1997), both based on low-dispersion or integrated indices, onto a scale fully based on high-dispersion spectroscopy. We give the functions required to transform $W(\mathrm{Ca}$ II $)$ and $[\mathrm{Fe} / \mathrm{H}]_{\mathrm{Zw}}$ values into our updated scale, superseding the previous calibration by Carretta \& Gratton (1997). 
We are grateful to Paolo Montegriffo for providing the $H S T$ and IRAC photometry of NGC 6528 prior to publication. We warmly thank Bernardo Salasnich for constructing for us the isochrones from the latest Padova models in advance of publication. J. G. C. and B. B. B. are grateful for partial support from NSF grant AST 98-19614. The entire Keck/HIRES user community owes a huge debt to Jerry Nelson, Gerry Smith, Steve Vogt, and many other people who have worked to make the Keck telescope and HIRES a reality and to operate and maintain the Keck Observatory. We are grateful to the W. M. Keck Foundation, and particularly its late president, Howard Keck, for the vision to fund the construction of the W. M. Keck Observatory. This research has made use of the SIMBAD database, operated at CDS, Strasbourg, France.

\section{APPENDIX}

\section{THE REDDENING OF NGC 6528}

Like other bulge clusters heavily reddened and/or affected by differential reddening, the value of $E(B-V)$ for NGC 6528 in the literature ranges over more than $0.2 \mathrm{mag}$, e.g., $0.46 \mathrm{mag}$ (Richtler et al. 1998) from $V$, I photometry, $0.55 \mathrm{mag}$ (Ortolani et al. 1992 and Zinn 1980) and 0.73 mag from cluster integrated colors (Reed, Hesser, \& Shawl 1988).

Since an underestimate of $0.05 \mathrm{mag}$ in the value of $E(B-V)$ translates into an underestimate of about 0.2 dex in metal abundance when temperatures are derived from colors (Cohen 1983), this issue deserves special care. Even methods such as that of Sarajedini (1994), which uses fits to the shapes of unreddened giant branches to derive metallicity and reddening simultaneously, are unreliable for very metal-rich clusters, since the most metal-rich of his calibrating clusters is 47 Tuc $([\mathrm{Fe} / \mathrm{H}]=-0.70)$.

With the present analysis, we have a spectroscopic determination of the temperatures of four RHB stars that are derived only from the excitation of many $\mathrm{Fe} \mathrm{I}$ lines. These estimates are therefore reddening free and provide an independent route to the value of $E(B-V)$ in NGC 6528.

As a starting point, we assumed a mean value of $T_{\text {eff }} \sim 4610 \mathrm{~K}$ as representative of the spectroscopic temperatures of our RHB stars (see Table 3). This temperature, with the mean metallicity and gravity from Table 3, corresponds to a value of $(V-K)_{0}=2.53$ based on the color- $T_{\text {eff }}$ calibration of Gratton, Carretta, \& Castelli (1996). ${ }^{9}$ On the other hand, from the $V, K$ photometry of P. Montegriffo (1999, private communication), the mean $V-K$ color for the four program stars is 3.88 ; so that, assuming a standard relation $E(V-K)=2.7 E(B-V)$ (see, e.g., Cardelli, Clayton, \& Mathis 1989), we deduce a value of $E(B-V)=0.50 \mathrm{mag}$ as an independent estimate for the reddening of NGC 6528. Repeating the above exercise using the individual colors and parameters of each star, we could not isolate any significant difference in the resulting values of $E(B-V)$.

This value is somewhat lower than the one listed in the compilation by Harris (1996), as well as most of the values quoted above, although only slightly smaller than that found in the field just north and west of the cluster by Stanek (1996). (Stanek's value in the field just south of the cluster is close to that of Harris.) We believe that this arises because of a selection bias. In a metal-rich globular cluster with differential reddening, the RHB shape in a CMD changes from the normal short horizontal one characteristic of a constant reddening to an extended distribution sloping toward fainter magnitudes for the redder stars. Such a RHB is clearly shown in the case of NGC 6553 in Figure $2 b$ of Ortolani, Barbuy, \& Bica (1990) and NGC 6528 (in Fig. 2). Our sample of RHB stars selected for high-dispersion spectroscopy is biased toward the brighter stars and hence the less reddened ones. Table 3 shows, however, that the program stars have a total range in $T_{\text {eff }}$ of $70 \mathrm{~K}$ (well within the adopted uncertainties), even though they cover a rather large range in the observed reddened CMD.

NGC 6528 is known to be affected by differential reddening. Cohen \& Sleeper (1995) establish that the range of $E(B-V)$ is about 0.25 mag. Consistent with the suggestion of differential reddening, it is interesting to note that three of the program stars are located in the same region of NGC 6528. For differential reddening of the magnitude found in NGC 6528, this selection effect far outweighs any tendency toward picking RHB stars that are slightly evolved off the zero-age horizontal branch.

Irrespective of the origin of this effect and of the mean value of $E(B-V)$ for NGC 6528, we stress here that the determination of temperatures by line excitation is a purely spectroscopic, reddening-free method, and that this is the method used for the four RHB stars we observed.

As noted by the referee, one may wonder how sensitive this method could be to errors in $g f$-values. In previous sections, we performed a test aimed to understand whether our values for the microturbulent velocity were biased because of the correlation existing between line strength and excitation potential, because lines of low excitation are all strong. Hence, we compared the values for the $v_{t}$ derived from all lines with those we derive using only high excitation lines. This second value is nearly independent of the assumed temperature because all lines have nearly the same excitation potential.

We checked the zero point of this calibration using field red clump stars (Carretta et al. 2001, Cohen et al. 2001) with atmospheric parameters similar to those of stars in NGC 6528 and with good parallaxes from Hipparcos. These stars are sufficiently close that it is reasonable to assume that the reddening is negligible.

\footnotetext{
${ }^{9}$ We do not have $B-V$ colors of the program stars, and we lack good $I$ photometry for the bright stars that were used to derive the zero-point correction to our color- $T_{\text {eff }}$ calibrations. Hence, the $V-I$ and $B-V$ colors do not add useful information to what we are deriving from $V-K$ colors.
} 
Armandroff, T. E., \& Da Costa, G. S. 1991, AJ, 101, 1329

Armandroff, T. E., \& Zinn, R. 1988, AJ, 96, 92

Barbuy, B., Renzini, A., Ortolani, S., Bica, E., \& Guarnieri, M. D. 1999, A\&A, 341, 539

Beaulieu, S. F., Gilmore, G., Elson, R. A. W., Johnson, R. A., Santiago, B., Sigurdsson, S., \& Tanvir, N. 2001, AJ, 121, 2618

Cardelli, J. A., Clayton, G. C., \& Mathis, J. S. 1989, ApJ, 345, 245

Carney, B. W. 1996, PASP, 108, 900

Carretta, E., Cohen, J. G., Gratton, R. G., \& Behr, B. B. 2001, in preparation

Carretta, E., \& Gratton, R. G. 1997, A\&AS, 121, 95

Cohen, J. G. 1983, ApJ, 270, 654

Cohen, J. G., Carretta, E., Gratton, R. G., \& Behr, B. B. 2001, in preparation

Cohen, J. G., Gratton, R. G., Behr, B. B., \& Carretta, E. 1999, ApJ, 523, 739 (Paper I)

Cohen, J. G., \& Sleeper, E. C. 1995, AJ, 109, 242

Cook, K., Mateo, M., Olszewski, E. W., Vost, S. S., Stubbs, C., \& Diercks, A. 1999, PASP, 111, 306

Côté, P. 1999, AJ, 118, 406

Côté, P., Mateo, M., Olszewski, E. W., \& Cook, K. H. 1999, ApJ, 526, 147

Davidge, T. J. 1999, astro-ph/9909408

Feast, M. W., Robertson, B. S. C., \& Black, C. 1980, MNRAS, 190, 227

Feltzing, S., \& Gilmore, G. 2000, A\&A, 355, 949

Gratton, R. G. 1987, MNRAS, 224, 175 .1989, A\&A, 208, 171

Gratton, R. G., et al. 2001, A\&A, 369, 87

Gratton, R. G., Carretta, E., \& Castelli, F. 1996, A\&A, 314, 191

Gratton, R. G., Carretta, E., Eriksson, K., \& Gustafsson, B. 1999, A\&A, 350,955

Gratton, R. G., \& Sneden, C. 1991, A\&A, 241, 501

Harris, W. E. 1996, AJ, 112, 1487

Ivans, I. I., Sneden, C., Kraft, R. P., Suntzeff, N. B., Smith, V. V., Langer, G. E., \& Fullbright, J. P. 1999, AJ, 118, 1273

Kraft, R. P., Sneden, C., Smith, G. H., Shetrone, M. D., \& Fulbright, J. 1998, AJ, 115, 1500

Kurucz, R. L. 1993a, CD-ROM 13, ATLAS9 Stellar Atmosphere Programs and $2 \mathrm{~km} / \mathrm{s}$ Grid (Cambridge: SAO)

\section{EFERENCES}

Kurucz, R. L. 1993b, CD-ROM 15, Diatomic Molecular Data for Opacity Calculations (Cambridge: SAO)

Kurucz, R. L., \& Bell, B. 1995, CD-ROM 23, Atomic Line Data (Cambridge:SAO)

Magain, P. 1984, A\&A, 134, 189

Mateo, M., Olszewski, E. W., Vogt, S. S., \& Keane, M. J. 1998, AJ, 116, 2315

McCarthy, J. K. 1988, Ph.D. thesis, Caltech

McWilliam, A., \& Rich, R. M. 1994, ApJS, 91, 749

Minniti, D. 1995, A\&AS, 113, 299

Ortolani, S. 1999, in The Chemical Evolution of the Galaxy: Stars versus Clusters, ed. F. Matteucci \& F. Giovanelli (Dordrecht: Kluwer), 301

Ortolani, S., Barbuy, B., \& Bica, E. 1990, A\&A, 236, 362

Ortolani, S., Bica, E., \& Barbuy, B. 1992, A\&AS, 92, 441

Ortolani, S., Renzini, A., Gilmozzi, R., Marconi, G., Barbuy, B., Bica, E., \& Rich, R. M. 1995, Nature, 377, 701

Reed, B. C., Hesser, J. E., \& Shawl, S. J. 1988, PASP, 100, 545

Rich, R. M. 1988, AJ, 95, 828

Rich, R. M., \& McWilliam, A. 2000, Proc. SPIE, 4005, 150

Richtler, T., Grebel, E. K., Subramaniam, A., \& Sagar, R. 1998, A\&AS, 127, 167

Rutledge, G. A., Hesser, J. E., \& Stetson, P. B. 1997a, PASP, 109, 907

Rutledge, G. A., Hesser, J. E., Stetson, P. B., Mateo, M., Simard, L., Bolte, M., Friel, E. D., \& Copin, Y. 1997b, PASP, 109, 883

Sadler, E. M., Rich, R. M., \& Terndrup, D. M. 1996, AJ, 112, 171

Salasnich, B., Girardi, L., Weiss, A., \& Chiosi, C. 2000, A\&A, 361, 1023

Sarajedini, A. 1994, AJ, 107, 618

Sharples, R., Walker, A., \& Cropper, M. 1990, MNRAS, 246, 54

Shortridge, K. 1988, FIGARO User's Manual (Starlink User Note 86) (version 2.4; Chilton: Rutherford Appelton Lab.)

Smith, G., \& Ragget, D. St. J. 1981, J. Phys. B, 14, 4015

Stanek, K. 1996, ApJ, 460, L37

Terndrup, D. M., Sadler, E. M., \& Rich, R. M. 1995, AJ, 110, 1774

Tonry, J. L., \& Davis, M. 1979, AJ, 84, 1511

Ventura, P., D'Antona, F., \& Gratton, R. G. 2001, ApJ, 550, L65

Vogt, S. E., et al. 1994, Proc. SPIE, 2198, 362

Zinn, R. 1980, ApJS, 42, 19

Zinn, R., \& West, M. J. 1984, ApJS, 55, 45 